\title{
REVIEW ARTICLE OPEN Theory and experimental evidence of phonon domains and their roles in pre-martensitic phenomena
}

\author{
Yongmei M Jin ${ }^{1}$, Yu U Wang ${ }^{1}$ and Yang Ren $^{2}$
}

Pre-martensitic phenomena, also called martensite precursor effects, have been known for decades while yet remain outstanding issues. This paper addresses pre-martensitic phenomena from new theoretical and experimental perspectives. A statistical mechanics-based Grüneisen-type phonon theory is developed. On the basis of deformation-dependent incompletely softened low-energy phonons, the theory predicts a lattice instability and pre-martensitic transition into elastic-phonon domains via 'phonon spinodal decomposition.' The phase transition lifts phonon degeneracy in cubic crystal and has a nature of phonon pseudo-Jahn-Teller lattice instability. The theory and notion of phonon domains consistently explain the ubiquitous pre-martensitic anomalies as natural consequences of incomplete phonon softening. The phonon domains are characterised by broken dynamic symmetry of lattice vibrations and deform through internal phonon relaxation in response to stress (a particular case of Le Chatelier's principle), leading to previously unexplored new domain phenomenon. Experimental evidence of phonon domains is obtained by in situ three-dimensional phonon diffuse scattering and Bragg reflection using high-energy synchrotron X-ray single-crystal diffraction, which observes exotic domain phenomenon fundamentally different from usual ferroelastic domain switching phenomenon. In light of the theory and experimental evidence of phonon domains and their roles in pre-martensitic phenomena, currently existing alternative opinions on martensitic precursor phenomena are revisited.

npj Computational Materials (2015) 1, 15002; doi:10.1038/npjcompumats.2015.2; published online 18 December 2015

\section{INTRODUCTION}

Pre-martensitic phenomena, also called martensite precursor effects, are long standing critical issues of martensitic phase transformation that have not been fully understood. Martensitic transformation is a typical solid-state displacive (diffusionless) phase transition that breaks crystal symmetry by development of spontaneous anisotropic lattice strain on cooling. ${ }^{1-4}$ Before martensitic transformation, the high-symmetry parent phase (cubic austenite) usually undergoes incomplete phonon softening in a wide temperature range $10-100 \mathrm{~K}$ above the martensite start temperature, ${ }^{4-6}$ which is accompanied by various anomalies that are unexpected in cubic phase. These precursor anomalies include diffuse scattering (streaks and satellites) in diffraction, crosshatched nanoscale striation image contrast (tweed patterns) in transmission electron microscopy (TEM), and anomalous thermal, acoustic, elastic properties (e.g., anisotropic thermal expansion, increased acoustic attenuation, frequency-dependent elastic moduli, etc.). ${ }^{4,7-14}$ Such pre-martensitic phenomena cannot be well explained from the existing phase transition theories. ${ }^{15,16}$ At the heart of the martensitic precursor problem is that these anomalies cannot be critical fluctuations before martensitic transformation because of the first-order nature of the martensitic transformation (a great majority of martensitic transformations are first-order phase transitions where certain phonon modes only exhibit incomplete softening thus soft-mode theory is inapplicable ${ }^{17}$ ), the high temperature range far beyond the vicinity of the transformation $(10-100 \mathrm{~K}$ above martensite start temperature), and the exceedingly long lifetime (much longer than TEM imaging time of tweed patterns). ${ }^{4,15}$ In particular, there exists a theoretical gap between the well-established experimental fact of incomplete phonon softening and the associated ubiquitous while puzzling martensitic precursor anomalies. To fill this gap and consistently explain these anomalies, a fundamental physical theory beyond a phenomenological model is needed, which may also predict previously unexplored new phenomena that can be tested by new experiments.

This paper addresses martensitic precursor phenomena from new theoretical and experimental perspectives. In the first part of this paper, a statistical mechanics-based phonon theory ${ }^{18}$ is discussed. The theory predicts a lattice instability and premartensitic transition into elastic-phonon domains via 'phonon spinodal decomposition,' and reveals the nature of this phase transition as phonon pseudo-Jahn-Teller lattice instability. The instability condition and the behaviours of phonon domains are discussed. Exotic domain phenomenon is predicted, which is fundamentally different from the usual ferroelastic domain switching phenomenon. In the second part of this paper, recent experimental evidence of phonon domains ${ }^{18}$ is presented. The theoretical predictions discussed in the first part of this paper are tested by specially designed experiments of in situ threedimensional (3D) phonon diffuse scattering and Bragg reflection using high-energy synchrotron X-ray single-crystal diffraction. In the third part of this paper, based on the theory and experimental evidence of phonon domains, the roles of phonon domains in pre-martensitic phenomena are discussed. The notion of phonon domains provides consistent explanations to the martensitic precursor 'anomalies' without resort to extrinsic defects, on the same physical footing of thermal expansion, both being intrinsic

\footnotetext{
${ }^{1}$ Department of Materials Science and Engineering, Michigan Technological University, Houghton, MI, USA and ${ }^{2}$ X-Ray Science Division, Advanced Photon Source, Argonne National Laboratory, Argonne, IL, USA.

Correspondence: YM Jin or YU Wang (ymjin@mtu.edu or wangyu@mtu.edu)

Received 8 July 2015; revised 13 August 2015; accepted 20 August 2015
} 
properties and natural consequences of anharmonic crystal lattices. The currently existing alternative explanations of the martensitic precursor anomalies (in particular, tweed patterns) are based on static defects (composition fluctuation, chemical disorder and point defects) that cause strain glass state in analogy to spin glass. ${ }^{15,19-24}$ These alternative opinions are revisited and examined against the new theoretical viewpoints and experimental results in light of the phonon domains and their roles in pre-martensitic phenomena.

\section{STATISTICAL MECHANICS-BASED PHONON THEORY OF PRE-MARTENSITIC TRANSITION}

A statistical mechanics-based phonon theory ${ }^{18}$ is recently developed to gain fundamental understanding of the effects of deformation-dependent low-energy phonons on the behaviours and properties of austenite before martensitic transformation. The theory is based on the experimentally established fact of incomplete phonon softening, which generally occurs in the cubic austenite in a wide temperature range 10-100 K above the martensite start temperature $\left(M_{\mathrm{S}}\right){ }^{4-6}$ The incomplete phonon softening phenomenon serves as the premise of the theory to derive consistent explanations to other pre-martensitic phenomena. Although the origin of incomplete phonon softening (also regarded as an anomaly-phonon anomaly ${ }^{25-28}$ ) is beyond the scope of the theory, it is believed to have an electronic origin. ${ }^{25,28}$ Since the same general phenomenon occurs in both nonmagnetic (e.g., $\mathrm{Ni}-\mathrm{Al}^{25,26}$ ) and magnetic (e.g., $\mathrm{Ni}-\mathrm{Mn}-\mathrm{Ga}^{27,28}$ ) systems, and both below and above Curie temperature $\left(T_{C}\right)$ in magnetic systems (e.g., $\mathrm{Ni}_{52.0} \mathrm{Mn}_{24.4} \mathrm{Ga}_{23.6}$ with $M_{\mathrm{S}}=308 \mathrm{~K}<T_{\mathrm{C}}=361 \mathrm{~K}$ and $\mathrm{Ni}_{53.0} \mathrm{Mn}_{26.4} \mathrm{Ga}_{20.6}$ with $M_{\mathrm{S}}=479 \mathrm{~K}>T_{\mathrm{C}}=368 \mathrm{~K}$ ), magnetism seems unlikely to have a general role (although magnetoelastic coupling $^{29}$ may have a certain role in magnetic materials), because a general mechanism identified in nonmagnetic systems would also work in magnetic systems. Figure 1 illustrates the incomplete phonon softening phenomenon as measured by inelastic neutron scattering ${ }^{27,30-32}$ and high-energy synchrotron $\mathrm{X}$-ray diffuse scattering, which is the premise for the following theoretical formulation.

Phonon-strain coupling and strain-dependent phonon frequencies Following Born's dynamical approach to crystal lattices, ${ }^{33}$ an effective potential function $\Phi$ of a cubic crystal is considered. Given the drastically distinct time scales of relatively slow lattice deformation and relatively fast thermal vibrations, $\Phi$ is expressed in terms of lattice strain $\varepsilon_{i j}$ (quasi-static configurational coordinates) and atomic displacements $u_{\alpha}^{\mathrm{r}}$ (dynamic vibrational coordinates) around average positions $\mathbf{r}$ of the lattice as defined by $\varepsilon_{i j}$. Taking into account the equilibrium condition of undeformed lattice and the invariance relations associated with lattice periodicity and rigid-body translation, ${ }^{33} \Phi\left(\varepsilon_{i j}, u_{a}^{r}\right)$ is expanded into Taylor series:

$$
\begin{aligned}
\Phi= & \Phi_{0}+\frac{1}{2} V C_{i j k l}^{0} \varepsilon_{i j} \varepsilon_{k l}+\frac{1}{2} \sum_{\mathbf{r}, \mathbf{r}^{\prime}} K_{a \beta}^{0}\left(\mathbf{r}-\mathbf{r}^{\prime}\right) u_{a}^{\mathbf{r}} u_{\beta}^{\mathbf{r}^{\prime}} \\
& +\frac{1}{2} \sum_{\mathbf{r}, \mathbf{r}^{\prime}} H_{a \beta i j}\left(\mathbf{r}-\mathbf{r}^{\prime}\right) \varepsilon_{i j} u_{a}^{\mathbf{r}} u_{\beta}^{\mathbf{r}^{\prime}}
\end{aligned}
$$

where $\Phi_{0}=\Phi\left(\varepsilon_{i j}=0, u_{a}^{\mathbf{r}}=0\right)$ is a constant and can be omitted, $C_{i j k l}^{0}=V^{-1} \partial^{2} \Phi / \partial \varepsilon_{i j} \partial \varepsilon_{k l}$ is adiabatic elastic modulus tensor, $V$ is crystal volume, $K_{\alpha \beta}^{0}\left(\mathbf{r}-\mathbf{r}^{\prime}\right)=\partial^{2} \Phi / \partial u_{a}^{\mathbf{r}} \partial u_{\beta}^{\mathbf{r}^{\prime}}$ is Born-von Kármán force constant matrix of the undeformed crystal, and $H_{a \beta i j}\left(\mathbf{r}-\mathbf{r}^{\prime}\right)=\partial^{3} \Phi / \partial u_{\alpha}^{\mathbf{r}} \partial u_{\beta}^{\mathbf{r}^{\prime}} \partial \varepsilon_{i j}$, where all partial derivatives are evaluated at $\varepsilon_{i j}=0$ and $u_{a}^{r}=0$, and summation convention over repeated indices is implied. The Taylor expansion is truncated after quadratic terms except the first nonvanishing coupling term between $\varepsilon_{i j}$ and $u_{a}^{r}$. Equation (1) can be rewritten in a form of perturbation:

$$
\Phi=\frac{1}{2} V C_{i j k l}^{0} \varepsilon_{i j} \varepsilon_{k l}+\frac{1}{2} \sum_{\mathbf{r}, \mathbf{r}^{\prime}}\left[K_{a \beta}^{0}\left(\mathbf{r}-\mathbf{r}^{\prime}\right)+\Delta K_{a \beta}\left(\mathbf{r}-\mathbf{r}^{\prime}, \varepsilon_{i j}\right)\right] u_{a}^{\mathbf{r}} u_{\beta}^{\mathbf{r}^{\prime}}
$$

where $\Delta K_{a \beta}\left(\mathbf{r}-\mathbf{r}^{\prime}, \varepsilon_{i j}\right)=H_{a \beta i j}\left(\mathbf{r}-\mathbf{r}^{\prime}\right) \varepsilon_{i j}$ is the perturbation to $K_{a \beta}^{0}\left(\mathbf{r}-\mathbf{r}^{\prime}\right)$ due to phonon-strain coupling. Equation (2) formulates a simple lattice dynamics problem of anharmonic crystal that contains the leading term of phonon-strain coupling, which leads to strain dependence of phonon frequencies that can be solved using perturbation method.

The first term in Equation (2) characterises the elastic energy of lattice deformation. The second term characterises the dynamics of lattice vibrations that takes into account the coupling between the vibrations and strain (anharmonicity). The eigen-frequencies $\omega_{\mathbf{k}, p}$ of normal mode vibrations (phonons) are determined by the secular equation $\left|\tilde{D}_{i j}(\mathbf{k}, \boldsymbol{\varepsilon})-\omega_{\mathbf{k}, p}^{2} \delta_{i j}\right|=0$, where $\tilde{D}_{i j}(\mathbf{k}, \boldsymbol{\varepsilon})=$ $\tilde{D}_{i j}^{0}(\mathbf{k})+\Delta \tilde{D}_{i j}(\mathbf{k}, \boldsymbol{\varepsilon})$ is the Fourier coefficients of the dynamical matrix $D_{i j}(\boldsymbol{\rho}, \boldsymbol{\varepsilon})=m^{-1} \mathrm{~K}_{i j}(\boldsymbol{\rho}, \boldsymbol{\varepsilon}), \quad \mathrm{K}_{i j}(\boldsymbol{\rho}, \boldsymbol{\varepsilon})=\mathrm{K}_{i j}^{0}(\boldsymbol{\rho})+\Delta \mathrm{K}_{i j}(\boldsymbol{\rho}, \boldsymbol{\varepsilon}), \quad m$ is atomic mass, and the phonon modes are labelled by wave vector $\mathbf{k}$ and polarisation vector $\mathbf{e}(\mathbf{k}, p)$. The eigen solutions of the secular equation $\left|\tilde{D}_{i j}^{0}(\mathbf{k})-\omega_{\mathbf{k}, p}^{2} \delta_{i j}\right|=0$ give the angular frequencies $\omega_{0}^{2}(\mathbf{k}, p)$ and polarisations $\mathbf{e}(\mathbf{k}, p)$ of the three phonon modes $(p=1,2,3)$ with wave vector $\mathbf{k}$ in the undeformed crystal. Incorporation of the perturbation term $\Delta \tilde{D}_{i j}(\mathbf{k}, \boldsymbol{\varepsilon})$ into the secular equation gives new eigen solutions where, to the first-order approximation, the polarisation vectors $\mathbf{e}(\mathbf{k}, p)$ remain the same while the eigen-frequencies become $\omega^{2}(\mathbf{k}, p)=\omega_{0}^{2}(\mathbf{k}, p)$ $+\Delta \omega^{2}(\mathbf{k}, p)$. The frequency change is given by

$$
\begin{aligned}
\Delta \omega^{2}(\mathbf{k}, p) & =\Delta \tilde{D}_{i j}(\mathbf{k}, \boldsymbol{\varepsilon}) e_{i}(\mathbf{k}, p) e_{j}(\mathbf{k}, p) \\
& =\Psi_{i j m n}(\mathbf{k}) e_{i}(\mathbf{k}, p) e_{j}(\mathbf{k}, p) \varepsilon_{m n}
\end{aligned}
$$

where $\Psi_{i j m n}(\mathbf{k})=m^{-1} \tilde{H}_{i j m n}(\mathbf{k})$ is the fourth-rank phonon-strain coupling coefficient tensor. Expanding $\Psi_{i j m n}(\mathbf{k})$ into Taylor series, taking into account the invariance with respect to $\mathbf{k} \leftrightarrow-\mathbf{k}$, and approximating the anisotropy in quadratic form yield $\Psi_{i j m n}(\mathbf{k})=\left[\Psi_{i j m n}^{0} \delta_{r s}+\frac{1}{2}\left(\partial^{2} \Psi_{i j m n} / \partial k_{r} \partial k_{s}\right) k^{2}\right] \kappa_{r} k_{s}=\Gamma_{r s i j m n}(k) k_{r} k_{s}$ where $\mathbf{K}=\mathbf{k} / \mathbf{k}$ is a unit vector along the wave vector $\mathbf{k}$. In terms of the sixth-rank phonon-strain coupling coefficient tensor $\Gamma_{r s i j m n}(k)$, the strain-dependent phonon frequency change becomes

$$
\Delta \omega^{2}(\mathbf{k}, p)=\Gamma_{r s i j m n}(k) \kappa_{r} K_{s} e_{i}(\mathbf{k}, p) e_{j}(\mathbf{k}, p) \varepsilon_{m n}
$$

The sixth-rank tensor $\Gamma_{r s i j m n}(k)$ complies with the crystal symmetry. Because of the three symmetry invariances with respect to $(r s) \leftrightarrow(s r),(i j) \leftrightarrow(j i)$ and $(m n) \leftrightarrow(n m)$ as required in Equation (4), $\Gamma_{\text {rsijmn }}$ has 216 independent components in its most general form, and a single-index Voigt notation $\Gamma_{a \beta \gamma}$ can be used for convenience with each index running from 1 to 6 (instead of the double-index tensor notation $\Gamma_{\text {rsijmn }}$ with each index running from 1 to 3). For cubic symmetry, there are only 12 independent components, namely, $\Gamma_{111}, \Gamma_{122}, \Gamma_{212}, \Gamma_{221}, \Gamma_{123}, \Gamma_{144}, \Gamma_{414}, \Gamma_{441}, \Gamma_{244}, \Gamma_{424}, \Gamma_{442}$ and $\Gamma_{456}$. (It is worth noting that, when all three indices in single-index Voigt notation (or three pairs of symmetric indices in double-index tensor notation) are interchangeable, such as in the case of the third-order elastic modulus tensor $C_{a \beta \gamma}$, the number of independent components of the sixth-rank tensor is further reduced to 6 for cubic symmetry, namely, $C_{111}, C_{122}, C_{123}, C_{144}, C_{244}$ and $C_{456}$.)

Deformation-dependent low-energy phonons of incompletely softened transverse acoustic [ $\left[\zeta_{0}\right]-\mathrm{TA}_{2}$ modes

As shown later in Equations (11a and 11b), , the effects of phononstrain coupling on the behaviours and properties of austenitic 

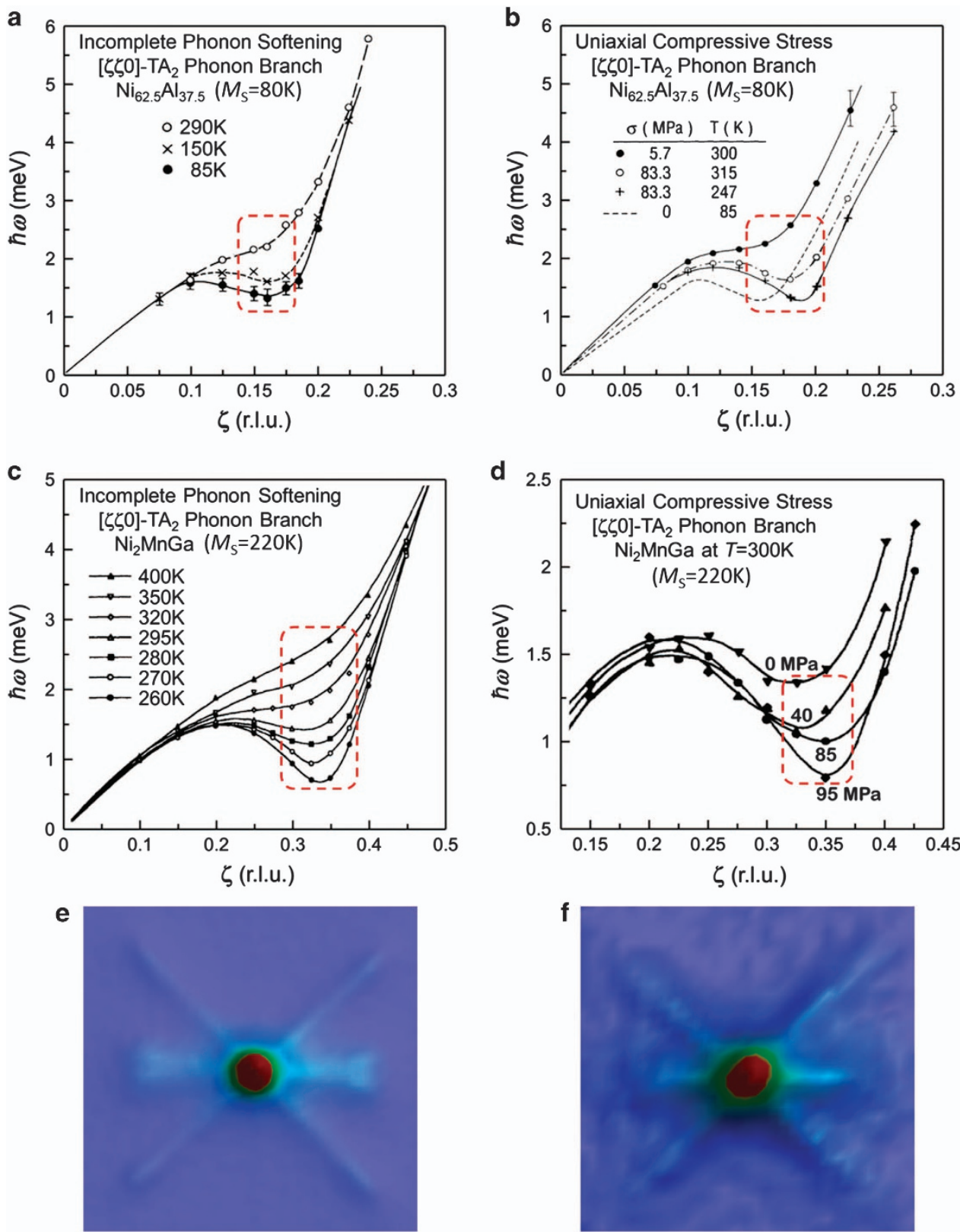

$\mathrm{Ni}_{52.0} \mathrm{Mn}_{24.4} \mathrm{Ga}_{23.6}$ at $T=310 \mathrm{~K}$ $\left(M_{\mathrm{S}}=308 \mathrm{~K}<T_{\mathrm{C}}=361 \mathrm{~K}\right)$

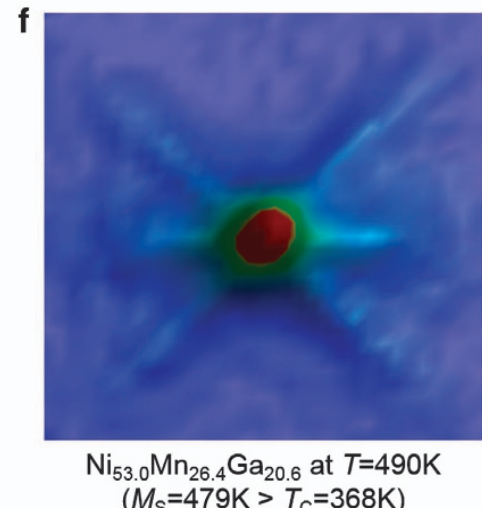

Figure 1. Incomplete phonon softening phenomenon and deformation-dependent low-energy phonons of transverse acoustic [ $\left.\zeta \zeta_{0}\right]-\mathrm{TA}_{2}$ branch in $(\mathbf{a}, \mathbf{b})$ nonmagnetic $\mathrm{Ni}_{62.5} \mathrm{Al}_{37.5}$ (refs 30,31) and (c, d) magnetic $\mathrm{Ni}_{2} \mathrm{MnGa}$ (refs 27,32) before martensitic transformations as measured by inelastic neutron scattering (reproduced with permission-refs 27,30-32). (a, c) Dependence on temperature. (b, d) Dependence on uniaxial compressive stress. Red dashed lines highlight specific phonon modes at the negative dip of dispersion curve that possess low energy and exhibit strongest dependence on stress. (e, f) Diffuse scattering from incompletely softened phonons of [ $\left.\zeta \zeta_{0}\right]-\mathrm{TA}_{2}$ branch before martensitic transformations as measured around (800) Bragg reflection peak using high-energy synchrotron X-ray single-crystal diffraction in (e) $\mathrm{Ni}_{52.0} \mathrm{Mn}_{24.4} \mathrm{Ga}_{23.6}$ with $M_{\mathrm{S}}=308 \mathrm{~K}<T_{\mathrm{C}}=361 \mathrm{~K}$ and (f) $\mathrm{Ni}_{53.0} \mathrm{Mn}_{26.4} \mathrm{Ga}_{20.6}$ with $M_{\mathrm{S}}=479 \mathrm{~K}>T_{\mathrm{C}}=368 \mathrm{~K}$.

crystals before martensitic transformation depend both on the phonon-strain coupling coefficients and on the phonon energies. It is the phonon modes with low energy and strong strain dependence that have the most significant role. To be specific,

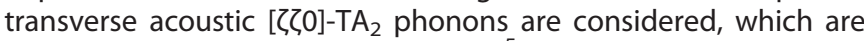
relevant to most martensitic systems. ${ }^{5}$ As illustrated in Figure 1, $[\zeta \zeta 0]-\mathrm{TA}_{2}$ phonon branch undergoes incomplete softening in austenite phases. ${ }^{27,30-32}$ In particular, the phonon modes corresponding to the negative dip in the dispersion curve at specific short wavelength, as highlighted by red dashed lines, possess low energy and exhibit the strongest dependence on stress (thus

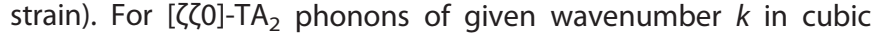
crystal, there are 12 degenerate phonon modes, namely, M1-M12 as listed in Table 1. Substituting the wave vector direction $\mathbf{к}$ and polarisation vector $\mathbf{e}$ of each phonon mode into Equation (4) gives the frequency change as a function of strain, as also listed in Table 1. It shows that the phonon frequencies depend on the strain only through 3 independent constants, which are combinations of the 12 independent components of the sixthrank phonon-strain coupling coefficient tensor $\Gamma_{r s i j m n}$, namely:

$$
a=\left(\Gamma_{111}+\Gamma_{122}+\Gamma_{123}+\Gamma_{212}+2 \Gamma_{221}-2 \Gamma_{441}-4 \Gamma_{442}\right) / 6
$$


Table 1. Twelve $[\zeta \zeta 0]-\mathrm{TA}_{2}$ phonon modes (M1-M12) of specific wavenumber $k$

Mode: $\sqrt{2} \mathbf{k} \mid \sqrt{2} \mathbf{e} \& \Delta \omega^{2}=\Gamma_{r s i j m n} k_{r} K_{s} e_{i} e_{j} \varepsilon_{m n}$ (note that $\Gamma$ is a function of $k$ )

$a=\left(\Gamma_{111}+\Gamma_{122}+\Gamma_{123}+\Gamma_{212}+2 \Gamma_{221}-2 \Gamma_{441}-4 \Gamma_{442}\right) / 6$

$b=\Gamma_{424}-\Gamma_{244}$

$c=-\left(\Gamma_{111}+\Gamma_{122}-2 \Gamma_{123}+\Gamma_{212}-\Gamma_{221}+4 \Gamma_{441}-4 \Gamma_{442}\right) / 12$

M1 and M2: $\pm[011] \mid[0 \overline{1} 1] \quad M 3$ and M4: $\pm[0 \overline{1} 1] \mid[011]$

$\Delta \omega^{2}=a \varepsilon_{V}+c\left(2 \varepsilon_{11}-\varepsilon_{22}-\varepsilon_{33}\right) \quad \Delta \omega^{2}=a \varepsilon_{V}+c\left(2 \varepsilon_{11}-\varepsilon_{22}-\varepsilon_{33}\right)-2 b \varepsilon_{23}$

$+2 b \varepsilon_{23}$

M5 and M6: $\pm[101] \mid[10 \overline{1}]$

$\Delta \omega^{2}=a \varepsilon_{V}+c\left(2 \varepsilon_{22}-\varepsilon_{33}-\varepsilon_{11}\right)$

$+2 b \varepsilon_{31}$

M9 and M10: $\pm[110] \mid[110]$

$\Delta \omega^{2}=a \varepsilon_{V}+c\left(2 \varepsilon_{33}-\varepsilon_{11}-\varepsilon_{22}\right)$

$+2 b \varepsilon_{12}$

M7 and M8: $\pm[10 \overline{1}] \mid[\overline{1} 0 \overline{1}]$

$\Delta \omega^{2}=a \varepsilon_{V}+c\left(2 \varepsilon_{22}-\varepsilon_{33}-\varepsilon_{11}\right)-2 b \varepsilon_{31}$

M11 and M12: $\pm[\overline{1} 10] \mid[\overline{110}]$

$\Delta \omega^{2}=a \varepsilon_{V}+c\left(2 \varepsilon_{33}-\varepsilon_{11}-\varepsilon_{22}\right)-2 b \varepsilon_{12}$

$$
\begin{aligned}
& b=\Gamma_{424}-\Gamma_{244} \\
& c=-\left(\Gamma_{111}+\Gamma_{122}-2 \Gamma_{123}+\Gamma_{212}-\Gamma_{221}+4 \Gamma_{441}-4 \Gamma_{442}\right) / 12
\end{aligned}
$$

It is readily seen from Table 1 that the symmetry-preserving volume strain $\varepsilon_{V}=\varepsilon_{11}+\varepsilon_{22}+\varepsilon_{33}$ contributes equally $\left(a \varepsilon_{V}\right)$ to all 12 modes thus, as expected, does not lift the phonon degeneracy. On the other hand, a symmetry-breaking strain changes the phonon frequencies differently for different modes, thus lifts the phonon degeneracy and leads to phonon energy level splitting among the 12 modes. The isotropic volume strain is responsible for thermal expansion as in Grüneisen model. ${ }^{34}$ The anisotropic deviatoric strain is responsible for the lifting of phonon degeneracy and the breaking of cubic lattice symmetry. Consider two examples of symmetry-breaking lattice deformation strain. Rhombohedral (trigonal) strain with trigonal axis along [111] is:

$$
\left[\varepsilon_{i j}\right]=\left[\begin{array}{ccc}
0 & \gamma / 2 & \gamma / 2 \\
\gamma / 2 & 0 & \gamma / 2 \\
\gamma / 2 & \gamma / 2 & 0
\end{array}\right]
$$

where isotropic volume strain has been separated out. Substituting Equation (6) into $\Delta \omega^{2}$ of the 12 phonon modes listed in Table 1 gives:

$$
\begin{aligned}
& \left.\begin{array}{c}
\omega_{1}^{2}=\omega_{0}^{2}+b \gamma \\
\omega_{\|}^{2}=\omega_{0}^{2}-b \gamma
\end{array}\right\} \quad \Rightarrow \\
& \left\{\begin{array}{l}
\omega_{1}=\omega_{2}=\omega_{5}=\omega_{6}=\omega_{9}=\omega_{10}=\omega_{1} \approx \omega_{0}+\frac{b \gamma}{2 \omega_{0}} \\
\omega_{3}=\omega_{4}=\omega_{7}=\omega_{8}=\omega_{11}=\omega_{12}=\omega_{\|} \approx \omega_{0}-\frac{b \gamma}{2 \omega_{0}}
\end{array}\right.
\end{aligned}
$$

Tetragonal strain with tetragonal axis along [001] and isotropic volume strain separated out is:

$$
\left[\varepsilon_{i j}\right]=\left[\begin{array}{ccc}
\eta / 3 & 0 & 0 \\
0 & \eta / 3 & 0 \\
0 & 0 & -2 \eta / 3
\end{array}\right]
$$

which gives:

$$
\begin{aligned}
& \left.\begin{array}{l}
\omega_{1}^{2}=\omega_{0}^{2}+c \eta \\
\omega_{\|}^{2}=\omega_{0}^{2}-2 c \eta
\end{array}\right\} \quad \Rightarrow \\
& \left\{\begin{array}{c}
\omega_{1}=\omega_{2}=\omega_{3}=\omega_{4}=\omega_{5}=\omega_{6}=\omega_{7}=\omega_{8}=\omega_{1} \approx \omega_{0}+\frac{c \eta}{2 \omega_{0}} \\
\omega_{9}=\omega_{10}=\omega_{11}=\omega_{12}=\omega_{\|} \approx \omega_{0}-\frac{c \eta}{\omega_{0}}
\end{array}\right.
\end{aligned}
$$

Grüneisen-type phonon theory of pre-martensitic transition A Grüneisen-type phonon theory is formulated in terms of the free energies of lattice deformation and quasi-harmonic phonons of a cubic crystal. ${ }^{18,35}$ For a crystal characterised by the effective potential function $\Phi\left(\varepsilon_{i j}, u_{a}^{r}\right)$ in Equation (2), the total free energy at temperature $T$ is a sum of lattice free energy $F_{\mathrm{L}}=E_{\mathrm{L}}-T S_{\mathrm{L}}$ and phonon free energy $F_{\mathrm{P}}=E_{\mathrm{P}}-T S_{\mathrm{P}}$. The entropy $S_{\mathrm{L}}$ describes the configurational entropy of a crystal lattice, whose change is negligibly small during diffusionless processes (such as elastic deformation and displacive transformation), thus can be regarded as a constant and omitted in isothermal analysis. Therefore, the lattice free energy is essentially the elastic energy $E_{\mathrm{L}}=\frac{1}{2} V C_{i j k l}^{0} \varepsilon_{i j} \varepsilon_{k l}$. The phonon energy $E_{\mathrm{p}}$ and entropy $S_{\mathrm{p}}$ are evaluated from Planck distribution of independent harmonic oscillators, which gives the free energy of each phonon mode $F_{\mathrm{P}(\mathbf{k}, p)}=\frac{1}{2} \hbar \omega_{\mathbf{k}, p}+k_{\mathrm{B}} T$ $\ln \left[1-\exp \left(-\hbar \omega_{\mathbf{k}, p} / k_{\mathrm{B}} T\right)\right]$, where $\hbar$ and $k_{\mathrm{B}}$ are reduced Planck constant and Boltzmann constant, respectively. The total free energy density (normalised by the crystal volume $V$ ) of lattice deformation and all phonon modes $\{\mathbf{k}, p\}$ is:

$$
F=\frac{1}{2} C_{i j k l}^{0} \varepsilon_{i j} \varepsilon_{k l}+\frac{k_{\mathrm{B}} T}{V} \sum_{\mathbf{k}, p} f\left(x_{\mathbf{k}, p}\right)
$$

where $x_{\mathbf{k}, p}=\hbar \omega_{\mathbf{k}, p} / k_{\mathrm{B}} T$ is dimensionless phonon energy and $f$ $(x)=x / 2+\ln \left(1-e^{-x}\right)$. As shown in Equation (4), the phonon frequencies are function of strain due to phonon-strain coupling, thus $x_{\mathbf{k}, p}=x_{\mathbf{k}, p}\left(\varepsilon_{i j}\right)$. The effects of the deformation-dependent phonons on the lattice stability is studied by analysing the derivatives of free energy with respect to strain:

$$
\begin{aligned}
& \frac{\partial F}{\partial \varepsilon_{i j}}=C_{i j k l}^{0} \varepsilon_{k l}+\frac{\hbar}{V} \sum_{\mathbf{k}, p} f^{\prime}\left(x_{\mathbf{k}, p}\right) \frac{\partial \omega_{\mathbf{k}, p}}{\partial \varepsilon_{i j}} \\
& \frac{\partial^{2} F}{\partial \varepsilon_{i j} \partial \varepsilon_{k l}}=C_{i j k l}^{0}+\frac{\hbar^{2}}{V k_{\mathrm{B}} T} \sum_{\mathbf{k}, p} f^{\prime \prime}\left(x_{\mathbf{k}, p}\right) \frac{\partial \omega_{\mathbf{k}, p} \partial \omega_{\mathbf{k}, p}}{\partial \varepsilon_{i j} \partial \varepsilon_{k l}}
\end{aligned}
$$

where linear dependence of phonon frequencies on strain is approximated as demonstrated in Equations (7) and (9). Equations (11a and 11b) show that both the phonon energies $\hbar \omega_{\mathbf{k}, p}$ and the phonon-strain coupling coefficients have important roles respectively through $f\left(x_{\mathbf{k}, p}\right)$ and $\partial \omega_{\mathbf{k}, p} / \partial \varepsilon_{i j}$.

As discussed in the section 'Deformation-dependent low-energy

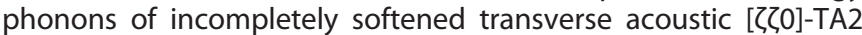
modes' and illustrated in Figure 1, the phonon modes corresponding to the negative dip in the dispersion curve at specific short wavelength possess both low energy and strong strain dependence thus have the dominant roles, which are the most significant terms in the sum in Equations (11a and 11b), i.e., $\sum_{\mathbf{k}, p}$ practically sums only over a small fraction of the first Brillouin zone. For simplicity without loss in conceptual generality, an Einstein-type model of density of states is adopted to focus on $N^{\prime}$ modes of such phonons ( $N^{\prime}<<3 N$, the total number of phonon modes), which are assumed to possess the same frequency and phonon-strain coupling coefficients in cubic crystal. To be specific,

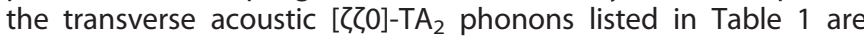
considered. The lattice stability is analysed with respect to two examples of lattice deformation. With respect to rhombohedral (trigonal) strain in Equation (6), the elastic energy becomes $E_{\mathrm{L}}=\frac{1}{6}\left(C_{11}^{0}+2 C_{12}^{0}\right) \varepsilon_{V}^{2}+\frac{3}{2} C_{44}^{0} \gamma^{2}$. According to whether the wave vector $\mathbf{k}$ is perpendicular to the trigonal [111] axis or not, the 12 $[\zeta \zeta 0]-\mathrm{TA}_{2}$ phonon modes of each given wavenumber $k$ fall into two groups, each group consisting of six modes with same frequency dependence on the strain, as shown in Equation (7). In this case, Equations (11a and $11 \mathrm{~b})$ yield

$$
\begin{aligned}
& \frac{\partial F}{\partial \varepsilon_{V}}=\frac{1}{3}\left(C_{11}^{0}+2 C_{12}^{0}\right) \varepsilon_{V}+\frac{3 \hbar a X}{2 \Omega \omega_{0}} f^{\prime}\left(x_{0}\right) \\
& \frac{\partial^{2} F}{\partial \gamma^{2}}=3 C_{44}^{0}+\frac{3 \hbar^{2} b^{2} X}{4 \Omega k_{B} T \omega_{0}^{2}} f^{\prime \prime}\left(x_{0}\right)
\end{aligned}
$$


where $\Omega=V / N$ is primitive cell volume, $X=N^{\prime} / 3 N$ and $x_{0}=\hbar \omega_{0} / k_{\mathrm{B}} T$. With respect to tetragonal strain in Equation (8), the elastic energy becomes $E_{\mathrm{L}}=\frac{1}{6}\left(C_{11}^{0}+2 C_{12}^{0}\right) \varepsilon_{V}^{2}+\frac{1}{3}\left(C_{11}^{0}-C_{12}^{0}\right) \eta^{2}$. According to whether the wave vector $\mathbf{k}$ is perpendicular to the tetragonal

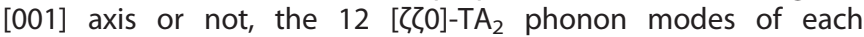
given wavenumber $k$ also fall into 2 groups, each group consisting of 8 and 4 modes respectively with same frequency dependence on the strain, as shown in Equation (9). In this case, Equations (11a and 11b) yield the same $\partial F / \partial \varepsilon_{V}$ in Equation (12) as expected and

$$
\frac{\partial^{2} F}{\partial \eta^{2}}=\frac{2}{3}\left(C_{11}^{0}-C_{12}^{0}\right)+\frac{3 \hbar^{2} c^{2} X}{2 \Omega k_{B} T \omega_{0}^{2}} f^{\prime \prime}\left(x_{0}\right)
$$

The isotropic volume strain $\varepsilon_{V}$ characterises the symmetrypreserving thermal expansion phenomenon (as in Grüneisen model $\left.^{34}\right)$ and is determined by $\left(\partial F / \partial \varepsilon_{V}\right)_{Y, \eta=0}=0$, which yields $\varepsilon_{V}^{0}=-9 \hbar a x f^{\prime}\left(x_{0}\right) / 2 \Omega \omega_{0}\left(C_{11}^{0}+2 C_{12}^{0}\right)$-note that $\varepsilon_{V}^{0}=\varepsilon_{V}^{0}\left[\omega_{0}(T), T\right]$ is temperature dependent. It is worth noting that $-a / 2 \omega^{2}$ corresponds to the Grüneisen parameter of an individual phonon mode, where the phonon-strain coupling constant $a$ is defined in Equation (5a). In fact, the sixth-rank phonon-strain coupling coefficient tensor $\Gamma_{\text {rsijmn }}$ extends the Grüneisen parameter from volume change of thermal expansion to anisotropic strain of symmetry-lifting displacive transformations. In particular, the phonon-strain coupling constants $b$ and $c$ defined in Equations ( $5 \mathrm{~b}$ and $5 \mathrm{c}$ ) respectively characterise the coupling of phonons with rhombohedral (trigonal) and tetragonal lattice deformation, as shown in Equations (7), (9) and (15a and 15b).

For the rhombohedral (trigonal) strain $\gamma$ and tetragonal strain $\eta$ characterising the symmetry-breaking anisotropic lattice deformation, $(\partial F / \partial \gamma)_{\gamma=0} \equiv 0$ and $(\partial F / \partial \eta)_{\eta=0} \equiv 0$ for cubic lattice. The lattice stability of cubic crystal is analysed through the second derivatives. The stability is lost against rhombohedral (trigonal) or tetragonal strain under the following respective conditions:

$$
\begin{aligned}
& \frac{\partial^{2} F}{\partial \gamma^{2}}=3\left[C_{44}^{0}+\frac{\hbar^{2} b^{2} X}{4 \Omega k_{\mathrm{B}} T \omega_{0}^{\prime 2}} f^{\prime \prime}\left(x_{0}^{\prime}\right)\right]<0 \\
& \frac{\partial^{2} F}{\partial \eta^{2}}=\frac{2}{3}\left[\left(C_{11}^{0}-C_{12}^{0}\right)+\frac{9 \hbar^{2} c^{2} X}{4 \Omega k_{\mathrm{B}} T \omega_{0}^{\prime 2}} f^{\prime \prime}\left(x_{0}^{\prime}\right)\right]<0
\end{aligned}
$$

where $\omega_{0}^{\prime}$ and $x_{0}^{\prime}$ incorporate the effect of thermal expansion, i.e., $\omega_{0}^{\prime}=\omega_{0}+a \varepsilon_{V}^{0} / 2 \omega_{0}$. Equations (15a and $\left.15 \mathrm{~b}\right)$ predict a premartensitic transition, on which the cubic symmetry of the crystal is spontaneously broken by anisotropic strain. The critical condition of the pre-martensitic transition is given by $\partial^{2} F / \partial \gamma^{2}=0$ and $\partial^{2} F / \partial \eta^{2}=0$ in the two exemplary cases, and is analysed via non-positive definiteness of $\partial^{2} F / \partial \varepsilon_{i j} \partial \varepsilon_{k l}$ in general. Figure 2a plots $f(x), f^{\prime}(x)$ and $f^{\prime \prime}(x)$. With decreasing $x=\hbar \omega / k_{\mathrm{B}} T, f^{\prime \prime}(x)$ rapidly approaches $-\infty$ and, as a result, realises the instability condition in Equations (15a and 15b). The decreasing energy of incompletely softened phonons with decreasing temperature, as illustrated in Figure 1, just satisfies the requirement for a pre-martensitic transition.

\section{'Phonon spinodal decomposition' and formation of phonon} domains

Equations (15a and 15b) describe a spinodal instability, which is related to the negative curvature (the second derivative) of the free energy, in analogy to spinodal decomposition. ${ }^{36,37}$ In particular, the Grüneisen-type phonon theory predicts the pre-martensitic transition to occur via 'spinodal decomposition' of phonon populations into phonon domains. The symmetrybreaking anisotropic strain changes the phonon frequencies differently for different modes, as given in Equation (4) and exemplified in Equations (7) and (9). Consequently, the thermal equilibrium occupation number of each phonon mode, $\bar{n}_{0}=\left[\exp \left(\hbar \omega_{0} / k_{B} T\right)-1\right]^{-1}$, changes according to its $\Delta \omega$ by an amount

$$
\Delta \bar{n}=-\frac{\Delta \omega \hbar \omega_{0}}{\omega_{0} k_{\mathrm{B}} T} \exp \left(\frac{\hbar \omega_{0}}{k_{\mathrm{B}} T}\right)\left[\exp \left(\frac{\hbar \omega_{0}}{k_{\mathrm{B}} T}\right)-1\right]^{-2}
$$

As illustrated in Figure $2 \mathrm{~b}$ for the case of rhombohedral (trigonal) deformation (tetragonal case is similar ${ }^{35}$ ), because $f^{\prime \prime}(x)<0$, the total phonon free energy decreases as a result of the changes in phonon frequencies (energies) and populations. Thus, the phonon populations have an intrinsic tendency to 'decompose' into phonon domains. Each phonon domain is a spatial region of dominant phonon mode with excess population, whose strength is characterised by $\Delta \bar{n} / \bar{n}_{0}$. The tendency of 'phonon spinodal decomposition' is, however, resisted by the elastic energy. Under the condition specified in Equations (15a and 15b), the decrease in phonon free energy outweighs the increase in elastic energy, leading to pre-martensitic transition.

The pre-martensitic transition breaks the cubic crystal symmetry and leads to formation of elastic domains. Elastic domains are characterised by the time-averaged static symmetry of crystal lattice, whose strength is described by the spontaneous anisotropic strain (e.g., $\gamma$ and $\eta$ ). The spontaneous strain of premartensitic phase is small as compared with the transformation strain of martensitic phase, and the pre-martensitic phase remains quasi-cubic. More importantly, the elastic domains are also phonon domains of redistributed phonon populations due to 'phonon spinodal decomposition.' Phonon domains are characterised by the broken dynamic symmetry of lattice vibrations, ${ }^{18,38}$ whose strength is described by the excess population of the dominant phonon mode with lowered phonon energy due to

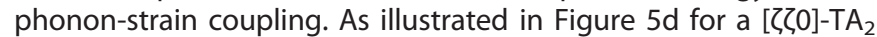
mode of wave vector $\mathbf{k}$ along [110] axis, the presence of such a dominant phonon mode with excess population in a lattice region (domain) produces anisotropic dynamic effects in the domain. Although the time-averaged atomic positions and thus the static symmetry of the local lattice are retained, the dynamic symmetry of the lattice vibration is broken due to the dominant phonon mode: the directions of wave vector $\mathbf{k}$ along [110], polarisation $\mathbf{u}$ along [1 $1 \overline{1} 0]$ and perpendicular $\mathbf{n}$ along [001] are inequivalent, and, in particular, the $\mathbf{n}$ direction along [001] is distinct from [100] and [010], which defines a dynamic tetragonal $\mathbf{n}$-axis. Consequently, the phonon domain exhibits anisotropic behaviours due to the broken dynamic symmetry of lattice vibrations. Furthermore, as discussed in the section 'Internal phonon relaxation in phonon domains and exotic domain phenomenon,' phonon domains can deform through internal phonon relaxation in response to applied stress. Such anisotropic phonon domains of broken dynamic symmetry would manifest themselves in phonon diffuse scattering under in situ stress (as observed by in situ synchrotron X-ray diffraction experiments discussed in the section 'Stress-dependent phonon diffuse scattering and Bragg reflection,' where Bragg reflection and phonon diffuse scattering together provide complementary information of crystal lattice and lattice vibrations). Therefore, the properties and behaviours of the elasticphonon domains are primarily characterised by the broken dynamic symmetry of lattice vibrations rather than the broken static symmetry of crystal lattice, in contrast to the conventional ferroelastic domains.

\section{Phonon pseudo-Jahn-Teller lattice instability}

Since the pre-martensitic transition via 'phonon spinodal decomposition' into phonon domains lifts the degeneracy of the phonons in cubic crystal, its nature can be interpreted as a phonon pseudo-Jahn-Teller lattice instability. It is caused by the interactions between fast lattice vibrations and slow lattice deformation (i.e., phonon-strain coupling), in analogy to JahnTeller instability that is caused by electron-phonon coupling 

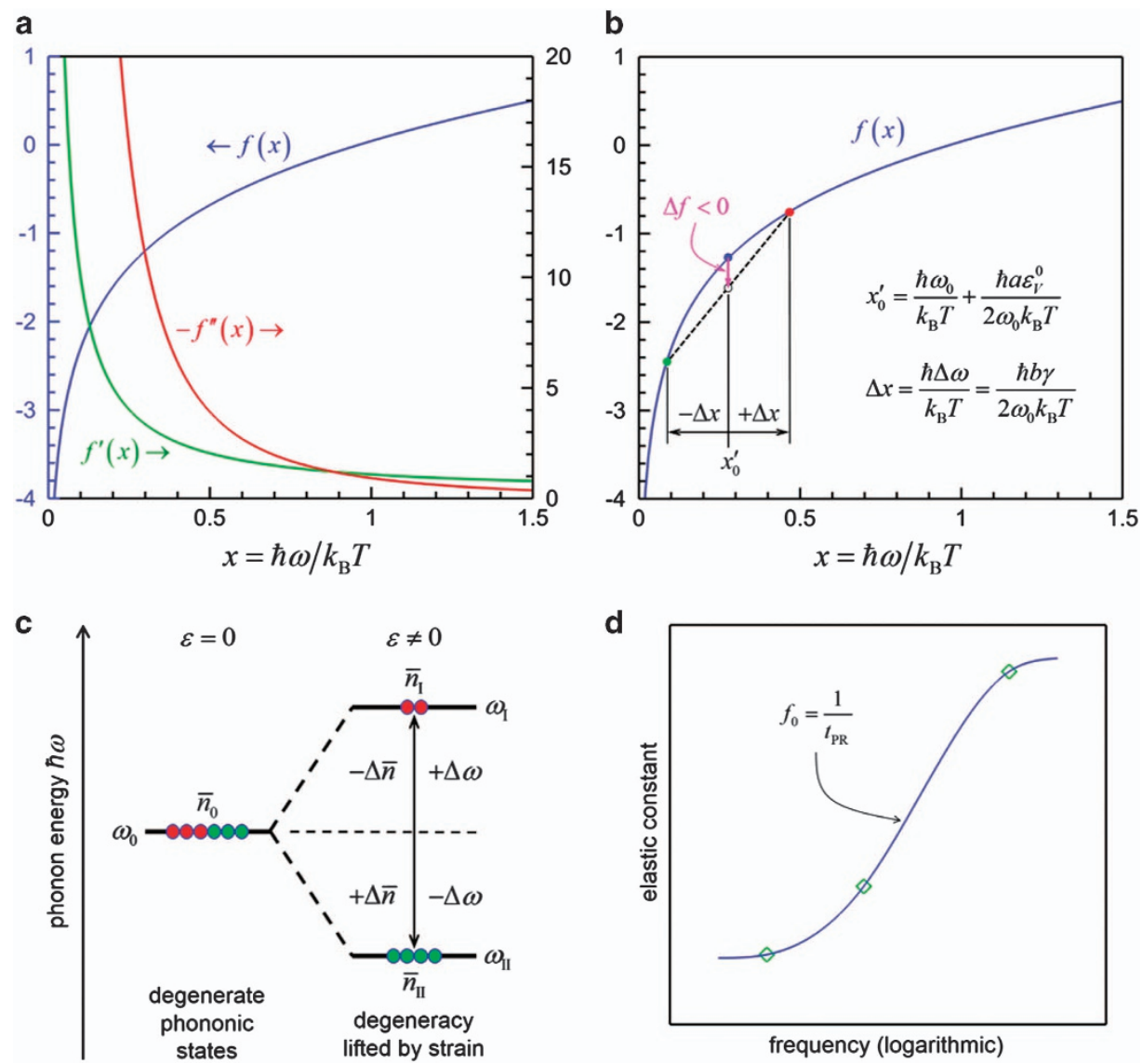

Figure 2. (a) Dependences of $f(x), f^{\prime}(x)$ and $f^{\prime \prime}(x)$ on $x=\hbar \omega / k_{\mathrm{B}} T$. (b) Illustration of pre-martensitic transition via 'phonon spinodal decomposition' associated with lattice deformation. (c) Schematic of phonon energy level splitting and phonon population redistribution due to lifting of phonon degeneracy by lattice strain. (d) Schematic of frequency dependence of elastic constant.

(the interactions between fast electronic and slow phononic processes). During the pre-martensitic transition, the crystal lattice spontaneously deforms to break the cubic symmetry and lift the phonon degeneracy, and in so doing lower the total phonon energy $E_{\mathrm{P}}=\sum_{\mathbf{k}, p}\left(1 / 2+\bar{n}_{\mathbf{k}, p}\right) \hbar \omega_{\mathbf{k}, p}$, where $\bar{n}_{\mathbf{k}, p}=\left[\exp \left(\hbar \omega_{\mathbf{k}, p} /\right.\right.$ $\left.\left.k_{\mathrm{B}} T\right)-1\right]^{-1}$. For cubic lattice, the above considered 12 equivalent

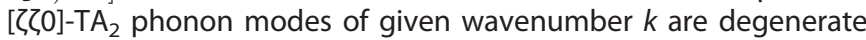
with the same frequency $\omega_{0}$ and equilibrium occupation number $\bar{n}_{0}$ for each mode. Such a degenerate phononic state is unstable due to phonon-strain coupling. For example, under rhombohedral (trigonal) lattice strain, the 12 phonon modes fall into 2 groups (6 in each) that undergo phonon energy level splitting: $\omega_{1}=\omega_{0}+\Delta \omega$ and $\omega_{\|}=\omega_{0}-\Delta \omega$, as shown in Equation (7) and illustrated in Figure 2c. Consequently, the phonon populations redistribute: $\bar{n}_{1}=\bar{n}_{0}-\Delta \bar{n}$ and $\bar{n}_{\|}=\bar{n}_{0}+\Delta \bar{n}$-note here $\Delta \bar{n}$ represents absolute value to explicitly emphasise the opposite sign to the frequency change magnitude $\Delta \omega$. Lifting phonon degeneracy lowers the total phonon energy: $\Delta E_{\mathrm{p}}=6 \hbar \omega_{\mid} \bar{n}_{1}+6 \hbar \omega_{\|} \bar{n}_{\|}-12 \hbar \omega_{0} \bar{n}_{0}=$ $-12 \hbar \Delta \omega \Delta \bar{n}<0$. Similar results can be demonstrated for tetragonal lattice strain. ${ }^{18}$ The phonon energy decrease $\Delta E_{\mathrm{p}} \propto-\Delta \omega \Delta \bar{n}$ is a general feature. Because $\Delta \omega \propto \varepsilon$ as shown in Equations (7) and (9) and $\Delta \bar{n} \propto \Delta \omega$ as shown in Equation (16), the phonon energy decrease is quadratically proportional to strain, $\Delta E_{\mathrm{p}} \propto-\varepsilon^{2}$, which competes with the elastic energy. With the effects of temperature and entropy taken into account, a statistical mechanics picture of strain-induced phonon energy level splitting and phonon population redistribution is equivalent to the thermodynamics picture of 'phonon spinodal decomposition.'

The phonon-strain pseudo-Jahn-Teller lattice instability exhibits some features distinct from the electron-phonon Jahn-Teller instability. Unlike electrons being fermions thus degenerate orbitals must be partially filled to produce Jahn-Teller instability, phonons are boson quasiparticles whose occupation numbers are free to change, thus phonon-strain coupling is a general mechanism of lattice instability. Moreover, the energy decrease in phonon pseudo-Jahn-Teller effect is quadratically proportional to strain (i.e., $\Delta E_{\mathrm{p}} \propto-\Delta \omega \Delta \bar{n} \propto-\varepsilon^{2}$ ), while in Jahn-Teller effect the energy decrease is linearly proportional to strain because the filling of lower-energy orbitals in ground state does not depend on the strain. Furthermore, the strength of the phonon pseudoJahn-Teller effect as measured by $\Delta E_{\mathrm{p}}$ depends not only on the phonon-strain coupling coefficients but also, more importantly, on the low phonon energy $\hbar \omega_{0}$. As the phonon energy decreases during incomplete phonon softening, the strength increases rapidly and eventually leads to the phonon pseudo-Jahn-Teller lattice instability and pre-martensitic transition.

Internal phonon relaxation in phonon domains and exotic domain phenomenon

Because the behaviours of the elastic-phonon domains are primarily characterised by the broken dynamic symmetry of lattice vibrations associated with the excess population of the dominant phonon mode, and the phonon populations redistribute in response to deformation strain due to phonon-strain coupling, phonon population redistribution provides an extra degree of freedom for internal relaxation of the domains in response to applied stress. Such internal phonon relaxation is a particular case of Le Chatelier's principle. ${ }^{34}$ According to Le Chatelier's principle, an external interaction with a system to disturb its internal equilibrium brings about internal processes to 


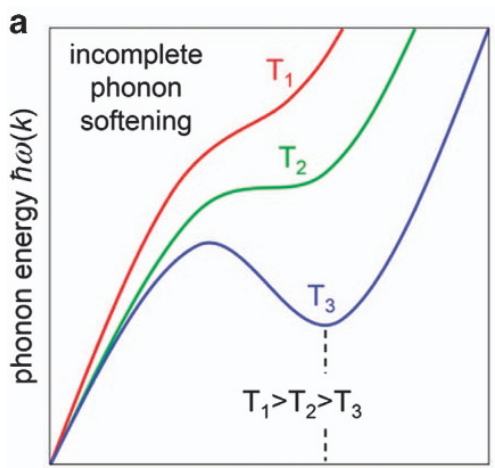

wavevector $k$

d

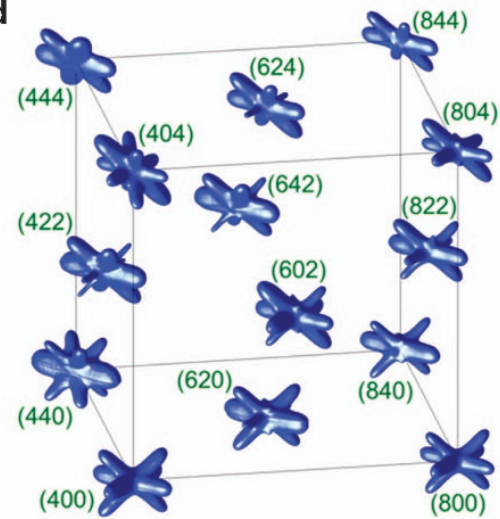

b

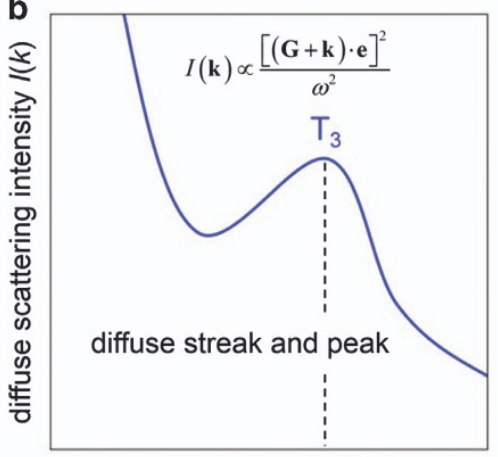

wavevector $k$

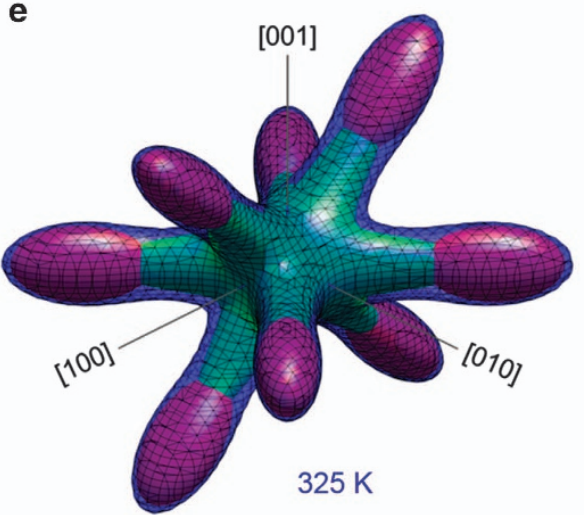

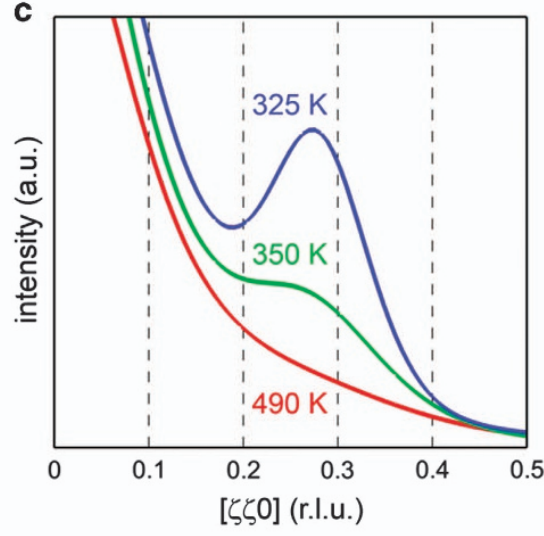

f

Figure 3. (a) Schematic of phonon dispersion curve at temperatures $T_{1}>T_{2}>T_{3}>M_{\mathrm{S}}$; a negative dip develops at $T_{3}$. (b) Schematic of intensity distribution of phonon diffuse scattering at $T_{3}$; a peak develops at the same wave vector of the dispersion dip. (c) Experimental measurement of temperature-dependent phonon diffuse scattering intensity distribution; a peak develops at $325 \mathrm{~K}$. (d) Experimental measurement of 3D phonon diffuse scattering from $<\zeta \zeta 0>-\mathrm{TA}_{2}$ modes around different Bragg reflections at $330 \mathrm{~K}$ without applied stress; extinction rule is determined by $(\mathbf{G} \cdot \mathbf{e})^{2}$. (e, f) Experimental measurement of 3D phonon diffuse scattering around (800) Bragg reflection at $325 \mathrm{~K}$ with satellite peaks and at $490 \mathrm{~K}$ without satellite peaks.

restore the equilibrium and reduce the effects of this interaction. In our case here, an externally imposed deformation disturbs the internal equilibrium of a pre-martensitic crystal and produces stress, which brings about internal relaxation processes via phonon population redistributions; on re-establishing the phonon populations into new thermal equilibrium, the stress response is reduced for the given externally imposed strain, leading to lower elastic modulus. In particular, it allows the pre-martensitic crystal to respond to applied stress via domain deformation rather than domain switching, in drastic contrast to the usual ferroelastic domain switching behaviour in martensitic phase. Thus, exotic domain phenomenon in pre-martensitic phase under external stress is expected from the perspective of phonon domains, where the exoticness is characterised by the following distinct features: domain deformation via internal phonon relaxation and phononstrain coupling without domain switching, reversibility and anhysteresis on unloading through thermal equilibration of phonon populations, and elasticity with low stiffness. As internal phonon relaxation takes time, the relaxation time of phonon population redistribution also leads to frequency dependence of the properties of pre-martensitic crystal. In particular, higher values of elastic constants are expected when measured by high-frequency technique (e.g., ultrasonic wave velocity) than by low-frequency technique (e.g., quasi-static stress-strain curve), as illustrated in Figure $2 \mathrm{~d}$, where a transition frequency $f_{0}$ corresponds to the reciprocal of phonon relaxation time $t_{\mathrm{PR}}$. Such frequency dependence offers a means to determine the characteristic time $t_{\mathrm{PR}}$ of internal phonon relaxation in the domains.

\section{EXPERIMENTAL EVIDENCE OF PHONON DOMAINS IN PRE-MARTENSITIC AUSTENITE}

Above theoretical predictions of phonon domains and their behaviours in austenite and pre-martensite phases are critically tested by specially designed experiments of in situ 3D phonon diffuse scattering and Bragg reflection using high-energy synchrotron X-ray single-crystal diffraction, ${ }^{18,38,39}$ which provide experimental evidence of phonon domains. Phonon diffuse scattering and Bragg reflection together provide complementary information of crystal lattice and lattice vibrations. High-flux highenergy synchrotron X-ray single-crystal diffraction is essential for such experimental tests ${ }^{39}$ : high energy provides deep penetration to probe bulk specimen, high flux enables short exposure time to measure weak diffuse scattering intensity that is critical for 3D reciprocal space mapping, single crystal allows loading along specific crystallographic axis, and 3D single-crystal diffraction allows measurement of diffuse scattering intensities from individual phonon modes-2D measurement using rocking crystal method causes intensity overlapping problem where scattering intensities from multiple phonon modes (such as $12<\zeta \zeta 0>-\mathrm{TA}_{2}$ branches) are all projected onto one image plate, as demonstrated in Figures $1 \mathrm{e}, \mathrm{f}$ and 7 . The diffuse scattering intensity from a phonon mode of wave vector $\mathbf{k}$, polarisation vector $\mathbf{e}$ and frequency $\omega$ around reciprocal lattice vector $\mathbf{G}$ (corresponding to Bragg reflection) is ${ }^{40}$ :

$$
I_{\mathbf{G}}(\mathbf{k}) \propto \frac{[(\mathbf{G}+\mathbf{k}) \cdot \mathbf{e}]^{2}}{\omega^{2}}
$$




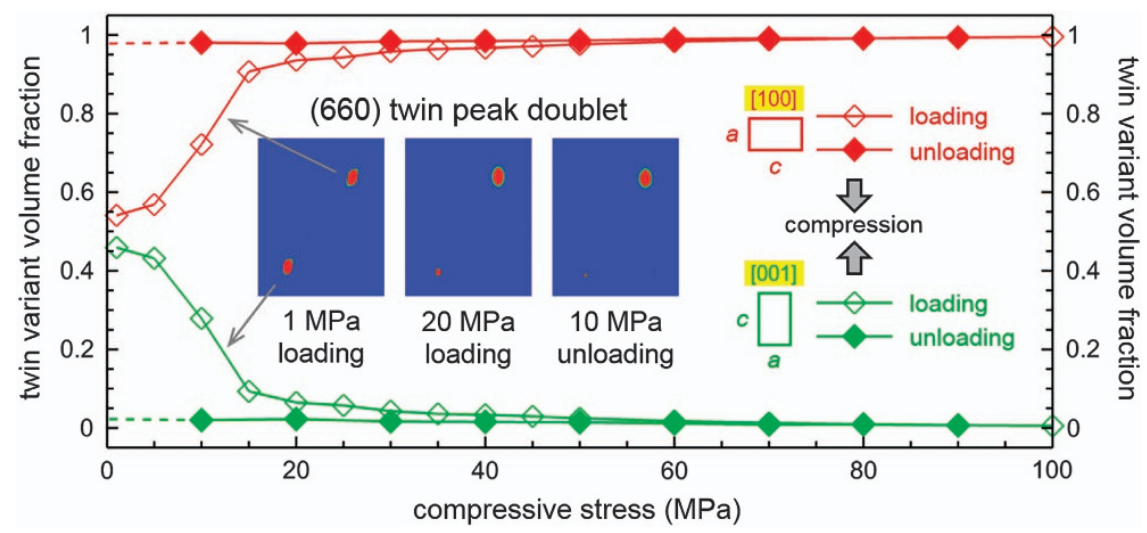

Figure 4. Evolutions of twin peak doublet of Bragg reflections and twin variant volume fractions during loading-unloading cycle in tetragonal martensite, showing usual ferroelastic domain switching (detwinning) and hysteresis.

a
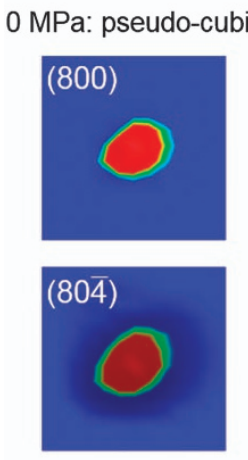

b

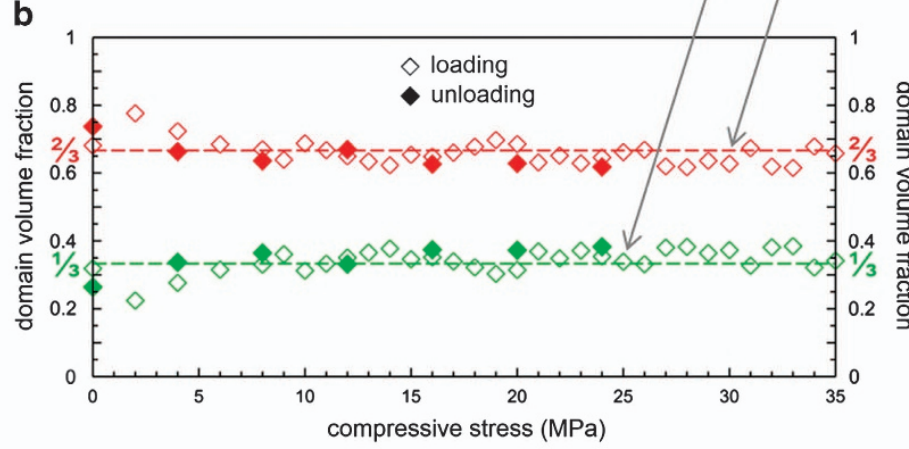

22 MPa: tetragonal
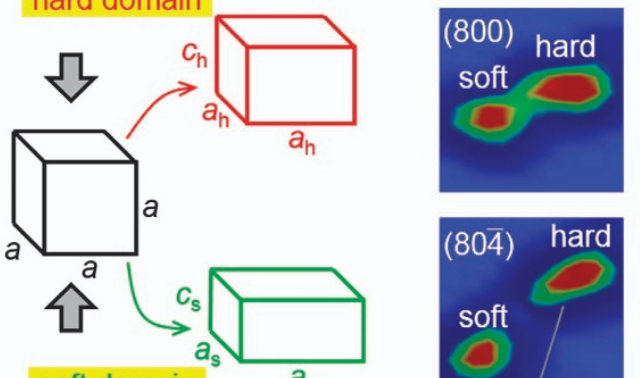

soft

.
C

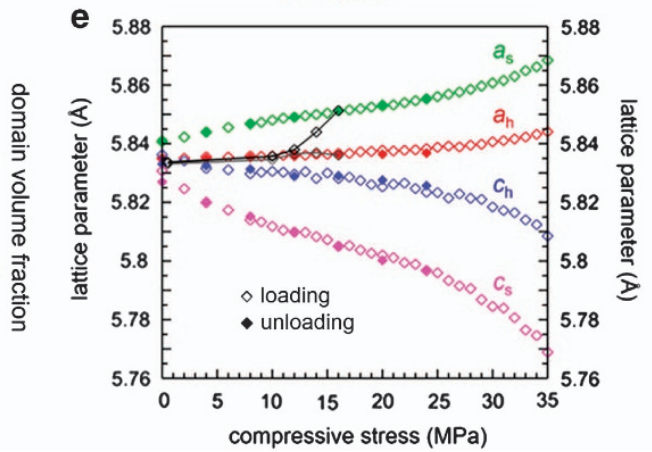

Figure 5. (a) Evolution of Bragg reflection peaks and (b) conservation of domain variant volume fractions during loading-unloading cycle in pre-martensite. (c) 12 [ $\zeta \zeta 0]-\mathrm{TA}_{2}$ phonon modes fall into two groups according to their tetragonal axes $\mathbf{n}$ with respect to [001] stress axis.

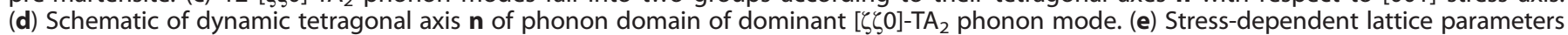
of hard (red, blue) and soft (green, pink) phonon domains as determined from Bragg peaks in (a). Lattice parameters (black) are also determined from the centres of 3D diffuse scattering in Figure $6 a-c$.

In light of phonon dispersion curves directly measured by inelastic neutron scattering as illustrated in Figure 1a-d, Equation (17) explicitly correlates the phenomena of phonon diffuse scattering and incomplete phonon softening (X-ray scattering has been used to determine phonon dispersions ${ }^{41}$ ). In particular, as illustrated in Figure $3 a, b$, when a negative dip develops at specific wave vector in the phonon dispersion curve during incomplete phonon softening, the intensity distribution of phonon diffuse scattering from this softened branch accordingly develops a peak at the same wave vector, as highlighted by dashed line. Thus, the diffuse streaks and satellites observed around Bragg reflections manifest the behaviours of the incompletely softened phonon modes. Together with Bragg reflection, phonon diffuse scattering is employed to study phonon domains in single crystals of
$\mathrm{Ni}_{49.90} \mathrm{Mn}_{28.75} \mathrm{Ga}_{21.35} \quad\left(M_{\mathrm{S}}=323 \mathrm{~K}, \quad T_{\mathrm{C}}=373 \mathrm{~K}, \quad\right.$ prepared by Adaptamat).

Temperature-dependent phonon diffuse scattering

The experimentally measured phonon diffuse scattering intensity distributions along wave vector $\mathbf{k}=[\zeta \zeta 0]$ (in reciprocal lattice unit, r.l.u.) around $\mathbf{G}=[800]$ at three representative temperatures are shown in Figure 3c. In agreement with the evolution of phonon dispersion curve during incomplete phonon softening as exemplified in Figure $1 a, c$ and with Equation (17), the diffuse streak becomes stronger on cooling, and diffuse satellite peak develops at low temperature (still above $M_{\mathrm{S}}$ ) manifesting a negative dip developed in the phonon dispersion curve. Shifting in the peak position to higher wave vector value with decreasing temperature 
a

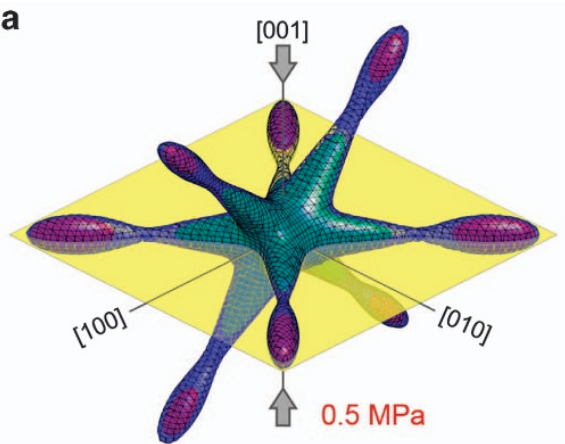

C

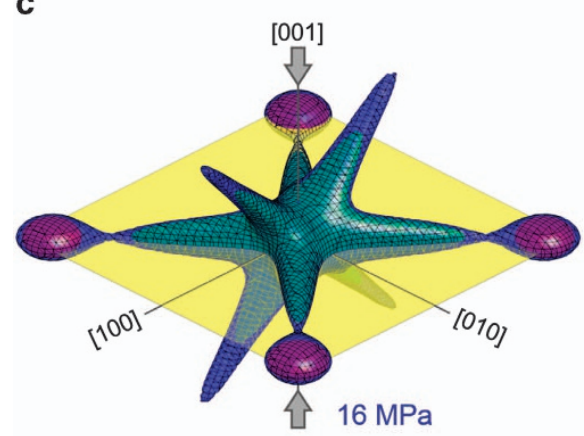

b

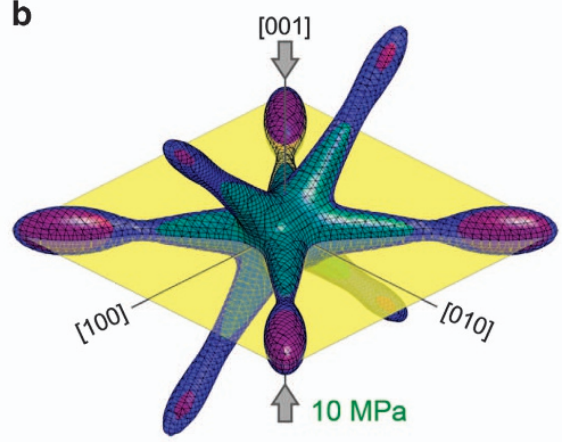

d

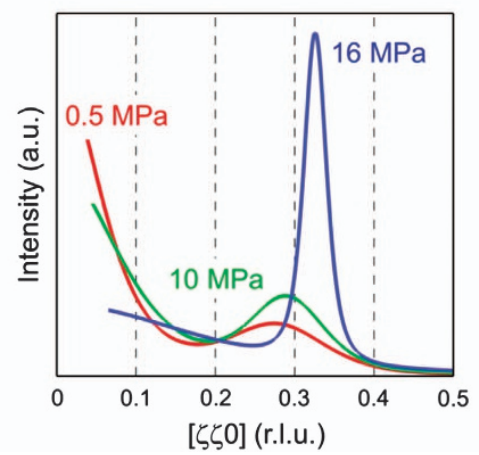

Figure 6. (a-c) Stress-dependent 3D phonon diffuse scattering around (800) Bragg reflection. (d) Stress dependence of diffuse scattering intensity distribution.

is also in agreement with the evolution of the negative dip position in the phonon dispersion curve. Figure 3e,f shows the experimentally measured 3D phonon diffuse scattering around (800) Bragg reflection, where satellite peaks are observed at $325 \mathrm{~K}$ while not at $490 \mathrm{~K}$. Figure $3 \mathrm{~d}$ shows the experimentally measured $3 \mathrm{D}$ phonon diffuse scattering around different Bragg reflections at $330 \mathrm{~K}$, where each diffuse rod originates from one of the $<\zeta \zeta 0\rangle-\mathrm{TA}_{2}$ phonon branches. As k.e $=0$ for transverse acoustic phonons, the phonon diffuse scattering exhibits systematic extinction around different Bragg reflections following the rule determined by (G.e) $)^{2}$ according to Equation (17). The observed phenomenon of temperature-dependent phonon diffuse scattering shown in Figure 3 agrees with the phenomenon of incomplete phonon softening.

Stress-dependent phonon diffuse scattering and Bragg reflection To contrast the exotic domain phenomenon predicted in premartensite to the usual ferroelastic domain switching phenomenon in martensite, $\mathrm{Ni}_{52.0} \mathrm{Mn}_{24.4} \mathrm{Ga}_{23.6}$ at $150 \mathrm{~K}$ is first studied, which transforms into nonmodulated tetragonal martensite below $220 \mathrm{~K}$. Figure 4 shows the evolution of Bragg reflection peak doublet from the twin-related martensite domain variants under loading-unloading cycle. The change in the peak intensities manifests the domain switching/detwinning process and allows determination of the twin variant volume fraction. The evolution of twin variant volume fraction as a function of stress exhibits large hysteresis on unloading, which is one important characteristic of usual ferroelastic domain switching phenomenon.

Figure $5 \mathrm{a}, \mathrm{b}$ shows the evolution of Bragg reflection peaks and conservation of domain variant volume fractions during loadingunloading cycle in pre-martensite of $\mathrm{Ni}_{49.90} \mathrm{Mn}_{28.75} \mathrm{Ga}_{21.35}$ at $350 \mathrm{~K}$ (above $M_{\mathrm{S}}=323 \mathrm{~K}$ ). Uniaxial stress is applied along single crystal [001] axis. Exotic domain phenomenon is observed from Bragg reflection, where the most unusual behaviour is that the domains deform into two (soft and hard) tetragonal lattices but do not switch. In particular, the volume fractions of soft and hard domain variants remain $1 / 3$ and $2 / 3$, respectively, during entire loading-unloading cycle. As illustrated in Figure $5 d$, the presence of dominant phonon modes in a domain breaks the dynamic symmetry of the lattice and makes the $\mathbf{n}$ direction a tetragonal axis. As shown in Equation (9) and illustrated in Figure 5c, according to whether their tetragonal axes $\mathbf{n}$ are parallel to the [001] stress axis or not (or equivalently, their wave vectors $\mathbf{k}$ perpendicular to the [001] stress axis or not), the $12[\zeta \zeta 0]-\mathrm{TA}_{2}$ phonon modes of each given wavenumber $k$ fall into 2 groups, each group consisting of 4 and 8 modes, respectively. As

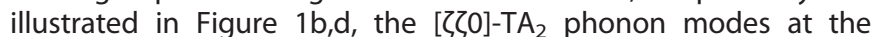
dispersion dip soften further in response to [001] stress, such internal phonon relaxation makes the 4 domains softer than the other 8 domains, leading to Bragg peak splitting under [001] stress shown in Figure $5 a$, and the $1 / 3$ and $2 / 3$ domain variant volume fractions manifest the $4: 8$ volume ratio of the soft and hard phonon domains. The values of lattice parameters $a_{1}$ and $a_{2}$ are slightly different under stress due to the anisotropy of the domains as illustrated in Figure $5 c$, resulting in anisotropic shape of the Bragg peak of the hard tetragonal lattice shown in Figure $5 \mathrm{a}$. Figure $5 \mathrm{e}$ shows the lattice parameters as function of stress, which exhibit reversibility free of hysteresis, indicating elastic deformation behaviours of both soft and hard domains. The lattice parameters $a_{\mathrm{s}[100]}$ and $a_{\mathrm{s}[001]}$ respectively along crystal [100] and [001] axes correspond to the elastically deformed soft domains with $\mathbf{k}$ along $\pm[110]$ or $\pm[1 \overline{1} 0]$, while $a_{\mathrm{h}[100]}$ and $a_{\mathrm{h}[001]}$ correspond to the hard domains with $\mathbf{k}$ along $\pm[011], \pm[01 \overline{1}]$, $\pm[101]$, or $\pm[10 \overline{1}]$. Although the hard phonon domains exhibit Young's modulus $E=14 \mathrm{GPa}$ and normal Poisson's ratio $v=0.33$, the soft domains exhibit significantly lower Young's modulus $E=4.8 \mathrm{GPa}$ and high Poisson's ratio $v=0.47$. The observed large, reversible, anhysteretic elastic strain response with low elastic stiffness agrees with the predictions from internal phonon relaxation and phonon-strain coupling.

The 3D phonon diffuse scattering around (800) Bragg reflection as function of stress shown in Figure $6 a-c$ reveals more information on the phonon domains. The in-plane diffuse scattering corresponding to $\mathbf{k}$ perpendicular to [001]-stress 
(k along $\pm[110]$ or $\pm[1 \overline{1} 0]$ within the yellow plane) grows in intensity with increasing [001] stress, while the out-of-plane diffuse scattering is depressed by the stress (note that only 4 diffuse rods with $\mathbf{k}$ along $\pm[101]$ or $\pm[10 \overline{1}]$ are present while the other 4 with $\mathbf{k}$ along $\pm[011]$ or $\pm[01 \overline{1}]$ are absent due to extinction). The enhanced in-plane diffuse scattering manifests the softening of the 4 phonon modes in the soft domains with tetragonal axis $\mathbf{n}$ along [001] stress axis. The in-plane diffuse satellites not only grow in intensity with increasing stress, but also concentrate into narrower and stronger peaks, and their incommensurate positions gradually shift towards $\zeta=1 / 3$, as shown in Figure $6 \mathrm{~d}$. These observations are in agreement with the evolution of phonon dispersion curve under stress as exemplified in Figure $1 b, d$. To confirm that each of the two tetragonal domains produces own phonon diffuse scattering, the lattice parameter $a$ is also determined respectively from the centres of in-plane and outof-plane diffuse scatterings around (800) Bragg peak, as also plotted in black symbols and lines in Figure $5 \mathrm{e}$. The out-of-plane diffuse scattering gives the same $a$ as $a_{\mathrm{h}[100]}$, indicating its association with the hard domains. The in-plane diffuse scattering gives $a$ that is close to $a_{\mathrm{h}[100]}$ at low stress and approaches $a_{\mathrm{s}[100]}$ with increasing stress, indicating its association with the soft domains. The transition from $a_{\mathrm{h}[100]}$ at low stress to $a_{\mathrm{s}[100]}$ with increasing stress is caused by the fact that the in-plane diffuse scattering is increasingly dominated by the soft phonon domains with increasing stress and the out-of-plane diffuse scattering is dominated by the hard phonon domains, while at low stress their diffuse scatterings overlap. Such exotic domain behaviours observed by combined 3D phonon diffuse scattering and Bragg reflection agree with the theoretical predictions and provide experimental evidence of phonon domains in pre-martensitic phase.

\section{THE ROLES OF PHONON DOMAINS IN PRE-MARTENSITIC PHENOMENA}

The notion of phonon domains formed by pre-martensitic transition via 'phonon spinodal decomposition' provides consistent explanations to the martensitic precursor 'anomalies' without resort to extrinsic defects. These 'anomalies' are shown to be natural consequences of incomplete phonon softening and are explained through the key roles played by phonon domains. In particular, the very existence of phonon domains makes the premartensitic austenite no longer homogeneous, which in and of itself explains various martensite precursor effects. As has been previously pointed out ${ }^{16}$ : 'The explanation of most of precursor effects is based on the existence of hetero-phase fluctuation in the parent phase. However, such fluctuation requires that the system climb over large energy barrier. It has been questioned whether such fluctuation is really possible.' The pre-martensitic transition produces heterogeneous structures in the pre-martensite phase consisting of phonon domains. It is worth noting that such premartensite is a thermodynamically equilibrium new phase rather than hetero-phase fluctuation in the parent phase. The currently existing alternative explanations of the pre-martensitic phenomena (in particular, tweed patterns) are based on static defects (composition fluctuation, chemical disorder and point defects) that induce martensite embryos and cause strain glass state in analogy to spin glass. ${ }^{15,19-24}$ In light of the theory and experimental evidence of phonon domains and their roles in pre-martensitic phenomena, ${ }^{18}$ these alternative opinions are revisited.

'Phonon spinodal decomposition' versus phonon condensation The pre-martensitic anomalies have been previously attributed to $[\zeta \zeta 0]-\mathrm{TA}_{2}$ phonon mode condensation into static displacement waves, resulting in lattice modulations. ${ }^{42}$ According to the
Grüneisen-type phonon theory, ${ }^{18}$ the pre-martensitic transition occurs via 'phonon spinodal decomposition' into phonon domains. With decreasing temperature, the phonons increasingly soften, and the lower phonon energy $x=\hbar \omega / k_{\mathrm{B}} T$ makes $f^{\prime \prime}(x)$ rapidly approach $-\infty$, which eventually fulfills the instability condition in Equations (15a and 15b) before the phonon energy softens to zero. It is important that such instability condition does not require phonon frequency to vanish, that is, soft mode is not required. In particular, the pre-martensitic transition takes place at incompletely softened phonon energy $\sim 1 \mathrm{meV}$, which corresponds to finite phonon frequency $\sim 1 \mathrm{THz}$. At such finite frequency, phonons 'decompose' into domains but do not condense into static displacement waves or lattice modulations. Thus, the pre-martensitic transition is fundamentally different from a soft-mode transition. Moreover, Equations (15a and 15b) imply that, even in the case of a soft mode whose frequency softens to zero on cooling, as long as there is phonon-strain coupling as characterised by nonzero coupling constants, the lattice instability will be reached and the pre-martensitic transition will occur before a soft-mode transition.

It is worth noting that the effects of phonon-strain coupling on the behaviours and properties of austenitic crystals before martensitic transformation depend not only on the phononstrain coupling coefficients $\Gamma_{r s i j m n}$ through $\partial \omega_{\mathbf{k}, p} / \partial \varepsilon_{i j}$ but also, more strongly, on the phonon energies $\hbar \omega_{\mathbf{k}, p}$ through $f\left(x_{\mathbf{k}, p}\right)$, as shown in Equations (11)-(15). The strong effect of incompletely softened phonon energy can be illustrated through $f^{\prime \prime}(x)$ from the experimental data shown in Figure $1 \mathrm{c}: \hbar \omega \approx 1.4 \mathrm{meV}$ at $295 \mathrm{~K}$ gives $f^{\prime \prime}(x) \approx-330$, which is enhanced to $f^{\prime \prime}(x) \approx-1,024$ for further softened phonon energy $\hbar \omega \approx 0.7 \mathrm{meV}$ at $260 \mathrm{~K}$. The phononstrain coupling coefficients have an electronic origin and, in principle, can be determined through $\omega_{\mathbf{k}, p}=\omega_{\mathbf{k}, p}\left(\varepsilon_{i j}\right)$ by firstprinciples density functional theory computations at finite temperature, e.g., using self-consistent $a b$ initio lattice dynamics approach. ${ }^{43}$ Such studies would identify the mechanisms of phonon-strain coupling and guide a search for materials with high phonon-strain coupling coefficients. Although it could be challenging to experimentally measure the phonon-strain coupling coefficients, the magnitude of the coefficients can be estimated from the stress-dependent phonon dispersion. For example, from the phonon dispersion curves in $\mathrm{Ni}_{2} \mathrm{MnGa}$ under [001] uniaxial compressive stress at $300 \mathrm{~K}$ shown in Figure $1 \mathrm{~d}$, it is estimated that $d(\hbar \omega) / d \sigma \approx 5 \mathrm{meV} / \mathrm{GPa}$ for the incompletely softened low-energy phonon modes at the dispersion dip (the modes that have the dominant roles in pre-martensitic transition). According to Equation (9) for tetragonal strain, $d \omega / d \varepsilon \approx c / \omega$, where the phonon-strain coupling constant $c$ is defined in Equation (5c). Using $d \omega / d \varepsilon=\hbar^{-1}[d(\hbar \omega) / d \sigma](d \sigma / d \varepsilon), \quad d \sigma / d \varepsilon \approx E \sim 10 \mathrm{GPa}$ and $\hbar \omega \sim 1 \mathrm{meV}$, it gives $c \sim 1 \times 10^{26} \mathrm{~s}^{-2}$. It would be meaningful to show that the lattice instability condition with respect to tetragonal strain shown in Equation (15b) is satisfied in pre-martensitic $\mathrm{Ni}-\mathrm{Mn}-\mathrm{Ga}$ crystals: using $T=300 \mathrm{~K}, \Omega \approx 25 \AA^{3}$ and $X \sim 0.01$, Equation (15b) becomes $C^{\prime}<5 \mathrm{GPa}$, where $C^{\prime}=\left(C_{11}^{0}-C_{12}^{0}\right) / 2$. The estimated value of $C^{\prime}$ agrees in order of magnitude with the experimental values from ultrasonic measurements. ${ }^{44}$ This condition indeed coincides with the general phenomenon of significantly reduced $C^{\prime}$ before martensitic transformation, ${ }^{5}$ which could be the reason that such phonon-driven effects appear as pre-martensitic phenomena or martensite precursor effects.

\section{Phonon diffuse scattering (streaks and satellites) versus superlattice reflection}

The diffuse scattering streaks have been previously attributed to strain diffuse scattering from static displacement field associated with martensite embryos, and the diffuse satellite spots have been regarded as superlattice reflection peaks thus interpreted as evidence of static displacement waves (periodic lattice 
a

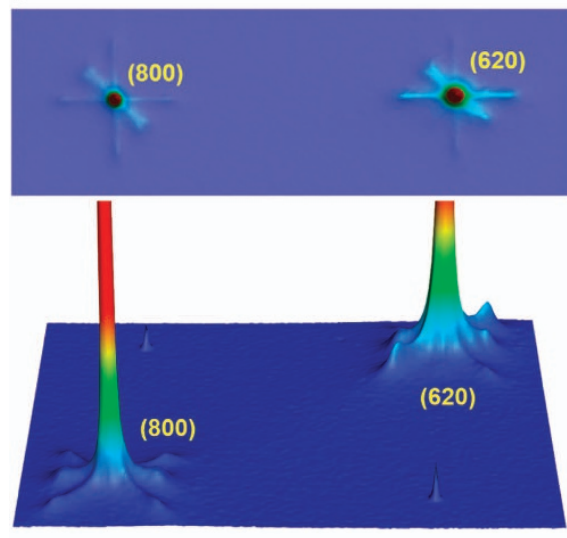

pre-martensitic austenite at $T=310 \mathrm{~K}$ b

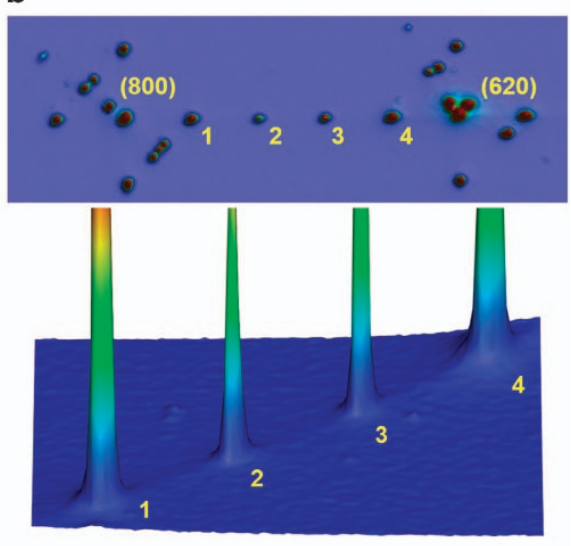

5-layered modulated martensite at $T=306 \mathrm{~K}$

Figure 7. Diffuse scattering and Bragg reflections from $\mathrm{Ni}_{52.0} \mathrm{Mn}_{24.4} \mathrm{Ga}_{23.6}\left(\mathrm{M}_{\mathrm{S}}=308 \mathrm{~K}\right)$ as measured by high-energy synchrotron $\mathrm{X}$-ray diffraction using single crystal rocking method. (a) Diffuse scattering from incompletely softened phonons of [ $\zeta \zeta 0]-\mathrm{TA}_{2}$ branch in pre-martensite at $2 \mathrm{~K}$ above $M_{\mathrm{S}}$. (b) Superlattice reflections from lattice modulations in 5-layered modulated martensite at $2 \mathrm{~K}$ below $M_{\mathrm{s}}$. Lower row shows $3 \mathrm{D}$ visualisations of the $2 \mathrm{D}$ intensities in upper row.

modulations). ${ }^{4,6-10,15,16,19-24,45}$ As shown in Equation (17), the diffuse scattering is directly related to the incomplete phonon softening and, in particular, manifests the shape of the phonon dispersion curve. When a phonon branch softens, it produces diffuse scattering streaks. When the phonon branch further softens on cooling, the diffuse streaks become stronger. When the phonon dispersion curve develops a negative dip at specific wave vector, the diffuse scattering produces satellite peaks accordingly around fundamental Bragg reflections. When the negative dip deepens on cooling, the diffuse satellite spots grow stronger. It is worth noting that the positions of the diffuse satellites correspond to the same wave vector of the negative dip in the phonon dispersion curve. In particular, the wave vector of both the negative dip and the diffuse satellites exhibits incommensurability to the cubic lattice and an evolution towards commensurability on cooling and/or stress loading. In the picture of static lattice modulations, the nature of the incommensurate lattice structure and its evolution in pre-martensitic phase have been puzzling. ${ }^{16}$ In light of phonon diffuse scattering, the incommensurate diffuse satellites correspond to the wave vector of the negative dip in the incompletely softened phonon dispersion curve, rather than the wave vector of lattice modulation waves.

It is also worth noting that, even from the viewpoint of superlattice reflections, the intensity profiles of the diffuse satellite peaks are highly unusual as compared with the normal superlattice reflection peaks. Figure 7 compares the satellite peak intensity profiles from pre-martensite phase and from modulated martensite phase. Modulated martensite usually produces arrays of well-defined strong satellite peaks from superlattice reflections with very weak or little diffuse streaks between them. On the other hand, pre-martensite produces weak diffuse peaks only around fundamental Bragg peaks, while there are no satellite arrays, and significant streaking intensity connects each diffuse peak to the fundamental Bragg peak. These features of diffuse scattering streaks and satellites in pre-martensite phase are difficult to explain by lattice modulations. The absence of satellite arrays implies that the lattice modulations assume perfect sine waveforms thus higher-order superlattice reflections have zero intensities, which is highly unusual for normal modulated phases. Moreover, to produce the diffuse streaks connecting to Bragg peaks, a spectrum of sinusoidal lattice modulations with increasing wavelengths is needed, which is also highly unusual for normal long-period structures. In light of phonon diffuse scattering, these features are manifestations of the shape of the incompletely softened phonon dispersion curve.

\section{Tweed patterns versus strain contrast}

The tweed patterns observed by TEM in pre-martensitic austenite have been previously attributed to static lattice displacements caused by point defects or martensite embryos. ${ }^{4,6,9,10,15,16,19-24,45}$ It is worth noting that, unlike twin that defines a crystallographic lattice configuration, 'tweed' is a term commonly used to describe a group of TEM images with qualitatively similar contrast features, i.e., cross-hatched nanoscale striations. A tradition to seek static structural origin of tweed patterns in martensitic precursor follows an early systematic TEM study of diffraction contrast from static shear strains associated with coherent precipitates (i.e., G.P. zones formed by atomic diffusion) in Cu-Be alloys. ${ }^{46}$ Owing to high elastic anisotropy, the elastic strains caused by tetragonal coherency of $\{100\}$ disc-shaped G.P. zones are resolved into $\{110\}\langle 110\rangle$ shears distributed in the matrix, which produce diffuse streaking, strain contrast and tweed patterns. The static displacements of $\{110\}\langle 1 \overline{1} 0\rangle$ shears have the same qualitative

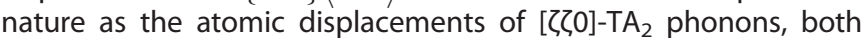
producing diffuse streaking along the same directions and exhibit the same systematic extinction rule. However, such strain diffuse scattering does not produce satellite peaks, while phonon diffuse scattering produces satellite peaks when dispersion curve develops a negative dip. Nevertheless, the diffuse scattering in both cases can be used to produce diffraction contrast in TEM to form tweed patterns. Thus, the tweeds of pre-martensite phase and coherent precipitation must be distinguished.

It is worth noting that only the phonons localised in the phonon domains can produce tweed patterns in TEM imaging. Travelling waves carry instantaneous image contrast to different regions while propagating through the specimen, thus averaging out the image contrast. In contrast, localised lattice vibrations within the phonon domains consistently produce diffraction contrast in the same lattice regions, producing tweed patterns. The tweed image contrast depends on the strength of diffuse scattering, which in turn depends on the degree of phonon softening. With decreasing temperature, the phonons soften further and the diffuse scattering becomes stronger, leading to increased diffraction image contrast of the tweed patterns.

Although the lattice strains caused and/or stabilised by extrinsic defects (composition heterogeneities, chemical disorders, point defects) may produce strain contrast and tweed patterns, they do 
not produce satellite peaks unless the defects order into superlattice structures characterised by the same wave vectors of the satellite peaks. Such defect arrangement is unlikely. Moreover, to explain the diffuse scattering satellites and their reversible evolutions with temperature and stress shown in Figures 3 and 6 , implausible ad hoc assumptions have to be made about ordered defect distribution and its reversible evolution and rapid response to stress, making defect-based explanations questionable. Furthermore, defects cannot explain the exotic domain phenomenon shown in Figure 5. In light of phonon diffuse scattering and phonon domains, these observations are naturally explained.

The pre-martensitic phase with phonon domains is a thermodynamically equilibrium state, which only depends on temperature and stress but not thermal-mechanical history, thus the observations shown in Figures 3, 5 and 6 are reproducible without exhibiting annealing effect. In addition, the tweed patterns are uniform throughout the crystal. In contrast, annealing effect is expected for defect-induced strain glass state, which is a thermodynamically nonequilibrium frozen state with random defects and uncorrelated local strain clusters in untransformed parent phase matrix. ${ }^{23}$ A nonequilibrium frozen strain glass state will evolve towards equilibrium on annealing, leading to history dependence. The experiments shown in Figures 3, 5 and 6 do not show annealing effect or history dependence in the single crystal samples used in the in situ diffraction.

\section{Pre-martensitic transition versus martensitic transformation}

There is a fundamental difference that distinguishes the 'phonon spinodal decomposition' pre-martensitic transition from a conventional martensitic transformation. The phonon dispersion in the cubic austenite phase and the pre-martensite phase exhibits a continuity in the dependence on temperature across the premartensitic transition. In contrast, there is a drastic discontinuous change in the phonon dispersion during martensitic transformation. The continuity of phonon dispersion as a function of temperature before martensitic transformation is illustrated in Figure $1 \mathrm{a}, \mathrm{c}$. The discontinuity of phonon dispersion across martensitic transformation is demonstrated in Figure 7. Although the incompletely softened phonons produce strong diffuse scattering in pre-martensite phase, the phonons become hard in martensite phase thus the diffuse scattering is very weak. The hard phonons in martensite phase imply that such 'phonon spinodal decomposition' and phonon-strain coupling do not have a role in martensite because these effects require low-energy softened phonons. It is worth noting that 'phonon spinodal decomposition' occurs at pre-martensitic transition and is characterised by the same phonon dispersion, while martensitic transformation is accompanied by drastic phonon spectrum reconstruction (indeed, displacive transformations are generally accompanied by phonon spectrum reconstruction resulting in different phonon contributions to the free energy of the phases). The nature of premartensitic transition has been previously treated as another martensitic transformation preceding the conventional one. ${ }^{47}$ In light of the phonon theory, the following new picture emerges: due to incomplete phonon softening in austenite phase, premartensitic transition naturally precedes martensitic transformation, where the former leads to elastic-phonon domains that are primarily characterised by broken dynamic symmetry of lattice vibrations while the latter leads to elastic domains that are primarily characterised by broken static symmetry of crystal lattice.

\section{Thermal, acoustic and elastic anomalies}

The anomalous thermal, acoustic and elastic properties are hard to understand for cubic austenite phase, which is expected to have a homogeneous structure without domains. The pre-martensitic transition produces heterogeneous structures in the premartensite phase consisting of elastic-phonon domains, which explains these anomalous effects. In particular, the anisotropic thermal expansion ${ }^{11,12}$ manifests the anisotropic behaviour of the elastic-phonon domains under the unavoidable mounting stress for dilatometer measurement. As shown in Figure 5, the elasticphonon domains are sensitive to stress, and small stress can induce significant strain due to the low elastic stiffness resulting from internal phonon relaxation. The increased acoustic attenuation $^{13}$ also results from the heterogeneous structures consisting of multiple elastic-phonon domains and the phonon relaxation processes. As discussed in the section 'Internal phonon relaxation in phonon domains and exotic domain phenomenon,' since the internal phonon relaxation takes time, it leads to frequency dependence of the elastic constants, where higher values are expected when measured at higher frequencies. Such frequency dependence has been observed in experiments. For example, in pre-martensitic $\mathrm{Ni}-\mathrm{Mn}-\mathrm{Ga}$ alloys, ultrasonic wave velocity measurement reports elastic modulus $\sim 150 \mathrm{GPa}^{44}$ while three-point bending measurement at $1 \mathrm{~Hz}$ reports elastic modulus $\sim 20 \mathrm{GPa}{ }^{48}$ Figure $2 \mathrm{~d}$ demonstrates three data points measured in In-TI respectively at $\sim 10 \mathrm{~Hz}, \sim 1 \mathrm{KHz}$ and $\sim 1 \mathrm{MHz} .{ }^{14}$ A systematic measurement of such kind would allow determination of the phonon relaxation time $t_{\mathrm{PR}}$ in the elastic-phonon domains through the transition frequency $f_{0}$. It is worth noting that these explanations are derived from the statistical mechanics-based phonon theory ${ }^{18}$ rather than a phenomenological model and do not resort to extrinsic defects.

\section{Fluctuation stabilisation and phonon localisation}

The Grüneisen-type phonon-strain coupling mechanism and the notion of phonon domains discussed above reveal additional aspects of the pre-martensitic phenomena, which are beyond the scope of this paper but worth brief discussions before concluding this paper. As mentioned in the 'Introduction' section, at the heart of the pre-martensitic problem, it has been thought that critical fluctuations before martensitic transformation could not have a significant role in the martensite precursor effects because of the first-order nature of the martensitic transformation and the high temperature range far above the vicinity of the transformation. Such an opinion must be changed in light of the 'phonon spinodal decomposition' pre-martensitic transition caused by phononstrain coupling. The Grüneisen-type phonon-strain coupling mechanism enhances the fluctuations before the pre-martensitic transition and stabilises the fluctuations in the pre-martensite phase, both at temperatures above the martensite start temperature, $M_{\mathrm{s}}$. In particular, at temperature right above the premartensitic transition temperature $\left(T_{\mathrm{PM}}\right)$, although the instability condition in Equations (15a and 15b) is not reached yet, the lattice stability has been greatly weakened by the phonon-strain coupling, thus heterogeneous fluctuations are significantly enhanced, which appear and disappear in the lattice thus the cubic symmetry is retained. At temperature below $T_{\mathrm{PM}}$ but still above $M_{S}$, these large heterogeneous fluctuations are stabilised by the phonon-strain coupling to form elastic-phonon domains, where the local cubic symmetry is broken. In this sense, the Grüneisen-type phonon-strain coupling is responsible for the entire pre-martensitic phenomena, where strongly developed heterogeneous fluctuations occur in a wide temperature range above $M_{s}$. Therefore, the pre-martensitic transition and its associated anomalous behaviours can be further investigated from the perspectives of phonon-strain coupling-enabled fluctuations and critical phenomena. ${ }^{34,49}$

The stabilisation of heterogeneous fluctuations into elasticphonon domains can be treated as phonon localisations due to phonon-strain coupling. It is worth noting that phonon localisation due to different mechanisms has been intensively studied theoretically and experimentally ${ }^{50-53}$ where, unlike the premartensitic phenomena discussed here, strain is not essential for 
phonon localisation. In perfect systems, intrinsic localised modes (also known as discrete breathers) form in discrete nonlinear systems, which are stable and do not involve extrinsic defects ${ }^{50,51}$ (note that perfect anharmonic crystals just possess the two critical components of intrinsic localised modes: nonlinearity and discreteness). In imperfect systems, Anderson localisation occurs in disordered systems, where interference effects of coherent scattering from multiple random scattering centres produce localised regions of trapped standing waves. ${ }^{52,53}$ In particular, Anderson localisation of ferroelectric transverse optical phonons has been employed to explain polar nanoregions in relaxor ferroelectrics, where nanoregions of standing ferroelectric phonons develop from preexisting resonance modes of Anderson localisation $^{53}$ (note that the Grüneisen-type phonon-strain coupling mechanism discussed in this paper involves ferroelastic transverse acoustic phonons). It would be intriguing to address the elastic-phonon domains from the perspectives of phonon localisation and standing waves. ${ }^{50,52}$

Finally, the notion of phonon domains addresses the premartensitic phenomena as intrinsic properties of perfect anharmonic crystal lattices caused by phonon-strain coupling without resort to extrinsic defects. In our phonon theory, both the phononstrain coupling and the incomplete phonon softening are treated as intrinsic properties of ideal austenitic crystals. First-principles studies employing supercells without extrinsic defects confirm such phonon behaviours as intrinsic properties. ${ }^{25,28,43}$ Although extrinsic defects are omnipresent in real materials and their effects undoubtedly exist, they are believed to have secondary roles through their interactions with the phonon domains. Understanding the effects of extrinsic defects in pre-martensitic transition is an important topic and deserves further investigations.

\section{CONCLUSION}

Martensitic precursor phenomena have been known for decades, while the puzzling anomalies are unexpected for cubic austenite phases above martensite start temperatures. They have been the topics of research workshops, conference symposia, review articles and book chapters $4,6,15,16,54,55$ (a large body of literature can be found in the references therein and in the proceedings of the conferences and workshops). This paper addresses martensitic precursor phenomena from new theoretical and experimental perspectives, and revisits and examines the currently existing alternative opinions in light of theory and experimental evidence of phonon domains and their roles in pre-martensitic phenomena.

Recent new theory and critical experiments are reviewed. A statistical mechanics-based phonon theory is developed by employing Born's dynamical approach to anharmonic crystal lattices. On the basis of a simple Taylor expansion of the effective potential function of anharmonic cubic crystal that contains the leading term of phonon-strain coupling, strain-dependent phonon frequencies are determined using perturbation method. A Grüneisen-type phonon theory is formulated in terms of the free energy of lattice deformation and quasi-harmonic phonons of the crystal, which predicts a loss of lattice stability and pre-martensitic transition into elastic-phonon domains via 'phonon spinodal decomposition.' The transition lifts the degeneracy of the phonons in cubic crystal thus has the nature of phonon pseudo-Jahn-Teller lattice instability. The predicted instability condition depends both on the phonon-strain coupling coefficients and, more strongly, on the phonon energies, thus the low energies of incompletely softened phonons in austenitic crystals before martensitic transformation just satisfy the requirement. The behaviours of the elastic-phonon domains are primarily characterised by the broken dynamic symmetry of lattice vibrations rather than the broken static symmetry of crystal lattice, in contrast to the conventional ferroelastic domains. As a particular case of Le
Chatelier's principle, phonon population redistribution in the elastic-phonon domains provides extra degree of internal relaxation in response to applied stress, which predicts exotic domain phenomenon in pre-martensitic austenite that is fundamentally different from the usual ferroelastic domain switching phenomenon in martensitic phase. The theoretical predictions are tested by specially designed experiments of in situ 3D phonon diffuse scattering and Bragg reflection using high-energy synchrotron X-ray single-crystal diffraction, which provide experimental evidence of phonon domains.

The roles of phonon domains in pre-martensitic phenomena are discussed, which provide consistent explanations to the martensitic precursor 'anomalies' on the same physical footing of thermal expansion, both being intrinsic properties and natural consequences of anharmonic crystal lattices. The incompletely softened phonons produce diffuse streaks and satellites in diffraction, and the dominant phonon modes localised in individual phonon domains produce diffraction contrast in TEM imaging of tweed patterns (not from the strain contrast of static displacements). The dependences of the diffuse satellite intensity and tweed image contrast on temperature are determined by the dependence of incomplete phonon softening on temperature as directly manifested in phonon dispersion curves. Other pre-martensitic 'anomalies' are also explained by the behaviours of phonon domains, e.g., the anisotropic thermal expansion manifests the anisotropic properties of the elastic-phonon domains, the increased acoustic attenuation results from the heterogeneous structures consisting of multiple elastic-phonon domains, and the frequency dependence of the elastic moduli is attributed to the internal relaxation process via the phonon population redistributions in response to external stress, which offers a means to determine the characteristic time of phonon relaxation. These explanations are derived from the statistical mechanics-based phonon theory rather than a phenomenological model and do not resort to extrinsic defects.

The theory is developed from the premise of incomplete phonon softening that is generally observed in both nonmagnetic and magnetic systems and both above and below Curie temperature. Although the origin of incomplete phonon softening is beyond the scope of the theory, it poses a question to future fundamental research. The theory adopts an Einstein-type model of density of states to focus on the deformation-dependent incompletely softened low-energy phonon modes that have dominant roles in pre-martensitic phenomena. Although such treatment can be improved by including all phonon modes if the phonon dispersion and phonon-strain coupling can be determined for individual modes, this simplification allows an illustration of the basic features determined only by symmetry and a few material parameters without loss of conceptual generality. This phonon theory fills a gap between the well-established experimental fact of incomplete phonon softening and the ubiquitous while puzzling martensitic precursor 'anomalies.' The theory can be extended to describe martensitic transformations. The current theory only considers the incompletely softened phonon modes that are characteristic of the cubic austenite and the premartensite phases. To describe martensitic transformation, it needs to incorporate the effective lattice potential, deformation and phonons of the martensite phase. Such further development would potentially lead to a unified phonon theory capable of describing the sequence of pre-martensitic, martensitic and intermartensitic phase transformations.

\section{ACKNOWLEDGEMENTS}

We are very grateful to Professor Armen G. Khachaturyan and Professor Long-Qing Chen for valuable discussions. Supports from NSF DMR-1409317 (YMJ) and DMR-1506936 (YUW) are acknowledged. Use of Advanced Photon Source at Argonne National Laboratory was supported by DOE DE-AC02-06CH11357. 


\section{COMPETING INTERESTS}

The authors declare no conflict of interest.

\section{REFERENCES}

1 Nishiyama, Z. Martensitic Transformation (Academic Press, 1978).

2 Roitburd, A. L. Martensitic transformation as a typical phase transformation in solids. Solid State Phys. 33, 317-390 (1978).

3 Khachaturyan, A. G. Theory of Structural Transformations in Solids (John Wiley \& Sons, 1983).

4 Salje, E. K. H. Phase Transitions in Ferroelastic and Co-elastic Crystals (Cambridge University Press, 1990).

5 Nakanishi, N. Elastic constants as they relate to lattice properties and martensite formation. Prog. Mater. Sci. 24, 143-265 (1980).

6 Planes, A. \& Mañosa, L. Vibrational properties of shape-memory alloys. Solid State Phys. 55, 159-267 (2001).

7 Shapiro, S. M., Noda, Y., Fujii, Y. \& Yamada, Y. X-ray investigation of the premartensitic phase in $\mathrm{Ni}_{46.8} \mathrm{Ti}_{50} \mathrm{Fe}_{3.2}$. Phys. Rev. B 30, 4314-4321 (1984).

8 Salamon, M. B., Meichle, M. E. \& Wayman, C. M. Premartensitic phases of $\mathrm{Ti}_{50} \mathrm{Ni}_{47} \mathrm{Fe}_{3}$. Phys. Rev. B 31, 7306-7315 (1985)

9 Oshima, R., Sugiyama, M. \& Fujita, F. E. Tweed structures associated with fcc-fct transformations in Fe-Pd alloys. Metall. Trans. A 19, 803-810 (1988).

10 Tanner, L. E., Schryvers, D. \& Shapiro, S. M. Electron microscopy and neutron scattering studies of premartensitic behavior in ordered Ni-Al $\beta_{2}$ phase. Mater. Sci. Eng. A 127, 205-213 (1990)

11 Liu, M., Finlayson, T. R., Smith, T. F. \& Tanner, L. E. Martensite precursor observations using thermal expansion: Ni-Al. Mater. Sci. Eng. A 157, 225-232 (1992).

12 Liu, M., Finlayson, T. R. \& Smith, T. F. Thermal expansion of $\mathrm{V}_{3} \mathrm{Si}$ with controlled martensite-phase morphology. Phys. Rev. B 52, 530-535 (1995).

13 Finlayson, T. R. Pretransformation phenomena as revealed by elastic waves. Metall. Trans. A 19, 185-191 (1988).

14 Wuttig, M. Unpublished experimental data in In-TI (private communication)

15 Krumhansl, J. A. Multiscale science: materials in the 21st century. Section 3 'Precursors: the next frontier'. Mater. Sci. Forum 327-328, 1-8 (2000).

16 Otsuka, K. \& Ren, X. Physical metallurgy of Ti-Ni-based shape memory alloys. Section "5. Precursor effects to martensitic transformations," in particular Section "5.9. Unsolved issues". Prog. Mater. Sci. 50, 511-678 (2005).

17 Krumhansl, J. A. \& Gooding, R. J. Structural phase transitions with little phonon softening and first-order character. Phys. Rev. B 39, 3047-3053 (1989).

18 Wang, Y. U \& Jin, Y. M. Martensitic transformation precursors: phonon theory and critical experiments. In: Militzer, $M$ et al. (eds). Proc Int Conf Solid-Solid Phase Transformations in Inorganic Materials 2015 (PTM 2015), Whistler, BC, Canada, 2015: 467-474. Full-version preprint at arXiv:1503.00027 (2015)

19 Semenovskaya, S. \& Khachaturyan, A. G. Kinetics of strain-related morphology transformation in $\mathrm{YBa}_{2} \mathrm{Cu}_{3} \mathrm{O}_{7-\delta}$. Phys. Rev. Lett. 67, 2223-2226 (1991).

20 Semenovskaya, S., Zhu, Y., Suenaga, M. \& Khachaturyan, A. G. Twin and tweed microstructures in $\mathrm{YBa}_{2} \mathrm{Cu}_{3} \mathrm{O}_{7-\delta}$ doped by trivalent cations. Phys. Rev. B 47, 12182-12189 (1993)

21 Kartha, S., Castán, T., Krumhansl, J. A. \& Sethna, J. P. Spin-glass nature of tweed precursors in martensitic transformations. Phys. Rev. Lett. 67, 3630-3633 (1991).

22 Kartha, S., Krumhansl, J. A., Sethna, J. P. \& Wickham, L. K. Disorder-driven pretransitional tweed pattern in martensitic transformations. Phys. Rev. B 52 803-822 (1995).

23 Sarkar, S., Ren, X. \& Otsuka, K. Evidence for strain glass in the ferroelastic martensitic system $\mathrm{Ti}_{50-x} \mathrm{Ni}_{50+x}$. Phys. Rev. Lett. 95, 205702 (2005).

24 Wang, D., Wang, Y., Zhang, Z. \& Ren, X. Modeling abnormal strain states in ferroelastic systems: the role of point defects. Phys. Rev. Lett. 105, 205702 (2010).

25 Zhao, G. L. \& Harmon, B. N. Phonon anomalies in $\beta$-phase $\mathrm{Ni}_{x} \mathrm{Al}_{1-x}$ alloys. Phys. Rev. B 45, 2818-2824 (1992).

26 Chou, H. \& Shapiro, S. M. Observation of predicted phonon anomalies in $\beta$-phase $\mathrm{Ni}_{50} \mathrm{Al}_{50}$. Phys. Rev. B 48, 16088-16090 (1993).

27 Zheludev, A. et al. Phonon anomaly, central peak, and microstructures in $\mathrm{Ni}_{2} \mathrm{MnGa}$. Phys. Rev. B 51, 11310-11314 (1995).

28 Bungaro, C., Rabe, K. M. \& Corso, A. D. First-principles study of lattice instabilities in ferromagnetic $\mathrm{Ni}_{2} \mathrm{MnGa}$. Phys. Rev. B 68, 134104 (2003).

29 Uijttewaal, M. A., Hickel, T., Neugebauer, J., Gruner, M. E. \& Entel, P. Understanding the phase transitions of the $\mathrm{Ni}_{2} \mathrm{MnGa}$ magnetic shape memory system from first principles. Phys. Rev. Lett. 102, 035702 (2009).
30 Shapiro, S. M., Yang, B. X., Shirane, G., Noda, Y. \& Tanner, L. E. Neutron scattering study of the martensitic transformation in a Ni-Al $\beta$-phase alloy. Phys. Rev. Lett. 62 , 1298-1301 (1989).

31 Shapiro, S. M., Svensson, E. C., Vettier, C. \& Hennion, B. Uniaxial-stress dependence of the phonon behavior in the premartensitic phase of $\mathrm{Ni}_{62.5} \mathrm{Al}_{37.5}$. Phys. Rev. B 48, 13223-13229 (1993).

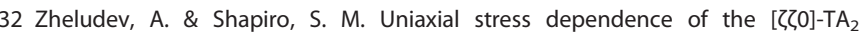
anomalous phonon branch in $\mathrm{Ni}_{2} \mathrm{MnGa}$. Solid State Commun. 98, 35-39 (1996).

33 Born, M. \& Huang, K. Dynamical Theory of Crystal Lattices (Oxford University Press, 1954).

34 Landau, L. D. \& Lifshitz, E. M. Statistical Physics (Pergamon Press, 1980).

35 Jin, Y. M. \& Wang, Y. U. Phonon theory of martensitic transformation precursors. Preprint at arXiv:1412.3725 (2014).

36 Cahn, J. W. On spinodal decomposition. Acta Metall. 9, 795-801 (1961).

37 Grimvall, G., Magyari-Köpe, B., Ozoliņš, V. \& Persson, K. A. Lattice instabilities in metallic elements. Rev. Mod. Phys. 84, 945-986 (2012).

38 Jin, Y. M. et al. Broken dynamic symmetry and phase transition precursor. Preprint at arXiv:1302.5479 (2013).

39 Cheng, T. L. et al. In-situ three-dimensional reciprocal-space mapping of diffuse scattering intensity distribution and data analysis for precursor phenomenon in shape-memory alloy. JOM 64, 167-173 (2012).

40 Warren, B. E. X-Ray Diffraction (Addison-Wesley Publishing, 1969).

41 Holt, M. et al. Determination of phonon dispersions from x-ray transmission scattering: the example of silicon. Phys. Rev. Lett. 83, 3317-3319 (1999).

42 Mañosa, L., Gonzàlez-Comas, A., Obradó, E. \& Planes, A. Anomalies related to the $\mathrm{TA}_{2}$-phonon-mode condensation in the Heusler $\mathrm{Ni}_{2} \mathrm{MnGa}$ alloy. Phys. Rev. B 55, 11068-11071 (1997).

43 Souvatzis, P., Eriksson, O., Katsnelson, M. I. \& Rudin, S. P. Entropy driven stabilization of energetically unstable crystal structures explained from first principles theory. Phys. Rev. Lett. 100, 095901 (2008).

44 Stipcich, M. et al. Elastic constants of Ni-Mn-Ga magnetic shape memory alloys. Phys. Rev. B 70, 054115 (2004).

45 Yamada, Y., Noda, Y., Takimoto, M. \& Furukawa, K. 'Modulated lattice relaxation' and incommensurability of lattice waves in $\beta$-based premartensitic phase. J. Phys. Soc. Jpn 54, 2940-2947 (1985).

46 Tanner, L. E. Diffraction contrast from elastic shear strains due to coherent phases. Philos. Mag. 14, 111-130 (1966).

47 Planes, A., Obradó, E., Gonzàlez-Comas, A. \& Mañosa, L. Premartensitic transition driven by magnetoelastic interaction in bcc ferromagnetic $\mathrm{Ni}_{2} \mathrm{MnGa}$. Phys. Rev. Lett. 79, 3926-3929 (1997).

48 Chernenko, V. A., Pons, J., Seguí, C. \& Cesari, E. Premartensitic phenomena and other phase transformations in Ni-Mn-Ga alloys studied by dynamical mechanical analysis and electron diffraction. Acta Mater. 50, 53-60 (2002).

49 PatashinskiĬ, A. Z. \& Pokrovskiĭ, V. L. Fluctuation Theory of Phase Transitions (Pergamon Press, 1979).

50 Campbell, D. K., Flach, S. \& Kivshar, Y. S. Localizing energy through nonlinearity and discreteness. Phys. Today 57, 43-49 (2004).

51 Manley, M. E., Abernathy, D. L., Agladze, N. I. \& Sievers, A. J. Symmetry-breaking dynamical pattern and localization observed in the equilibrium vibrational spectrum of Nal. Sci. Rep. 1, 4 (2011).

52 Lagendijk, A., van Tiggelen, B. \& Wiersma, D. S. Fifty years of Anderson localization. Phys. Today 62, 24-29 (2009).

53 Manley, M. E. et al. Phonon localization drives polar nanoregions in a relaxor ferroelectric. Nat. Commun. 5, 3683 (2014).

54 Tanner, L. E. \& Soffa, W. A. Pretransformation behavior related to displacive transformations in alloys: foreword. Metall. Trans. A 19, 760 (1988).

55 Tanner, L. E. \& Wuttig, M. Workshop on first-order displacive phase transformations: review and recommendations. Mater. Sci. Eng. A 127, 137-144 (1990).

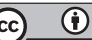

This work is licensed under a Creative Commons Attribution 4.0 International License. The images or other third party material in this article are included in the article's Creative Commons license, unless indicated otherwise in the credit line; if the material is not included under the Creative Commons license, users will need to obtain permission from the license holder to reproduce the material. To view a copy of this license, visit http://creativecommons.org/licenses/ by/4.0/ 